\title{
HILBERT MODULAR FORMS WITH PRESCRIBED RAMIFICATION
}

\author{
JARED WEINSTEIN
}

\begin{abstract}
Let $K$ be a totally real field. We present an asymptotic formula for the number of Hilbert modular cusp forms $f$ with given ramification at every place $v$ of $K$. When $v$ is an infinite place, this means specifying the weight of $f$ at $k$, and when $v$ is finite, this means specifying the restriction to inertia of the local Weil-Deligne representation attached to $f$ at $v$. Our formula shows that with essentially finitely many exceptions, the cusp forms of $K$ exhibit every possible sort of ramification behavior.
\end{abstract}

\section{Introduction and Main Theorem}

Let $K$ be a totally real field. Our investigation is concerned with counting the number of cuspidal automorphic representations $\pi=\otimes_{v} \pi_{v}$ of the adele group $\mathrm{GL}\left(2, \mathbf{A}_{K}\right)$ whose local components $\pi_{v}$ have prescribed ramification for all places $v$ of $K$. We must explain what is meant by "prescribed ramification": When $v$ is an infinite place, it means that $\pi_{v}$ is a prescribed essentially discrete series representation of $\operatorname{GL}(2, \mathbf{R})$ When $v$ is a finite place, it means that the Weil-Deligne representation associated to $\pi_{v}$ under the local Langlands correspondence has prescribed restriction to inertia and monodromy operator.

Our problem may be restated in terms of the $\ell$-adic Galois representations attached to Hilbert modular eigenforms over $K$. Suppose $f$ is such a form with coefficients in $\overline{\mathbf{Q}}_{\ell}$. Then there is a corresponding $\ell$ adic Galois representation $\rho_{f}: \operatorname{Gal}(\bar{K} / K) \rightarrow \mathrm{GL}\left(2, \overline{\mathbf{Q}}_{\ell}\right)$, as in [Tay89]. Fix an $\ell$ and suppose the following data are given:

(1) For each infinite place $v$, a weight $k_{v} \geq 2$, and

(2) For each finite place $v$ not dividing $\ell$, a representation $\sigma_{v}: I_{K_{v}} \rightarrow$ $\mathrm{GL}\left(2, \overline{\mathbf{Q}}_{\ell}\right)$ of the inertia group $I_{K_{v}}$ which extends to the full Galois group $\operatorname{Gal}\left(\bar{K}_{v} / K_{v}\right)$. Assume almost all of these are trivial.

We are then concerned with counting the number of Hilbert modular forms $f$ of weights $\left(k_{v}\right)$ and level prime to $\ell$ for which the restriction of $\rho_{f}$ to $I_{K_{v}}$ is $\sigma_{v}$ for each finite place $v$. Our main Theorem 1.1 gives an asymptotic formula for the number of such forms. Barring a natural 
obstruction coming from the central character, it shows that such forms always exist unless the data $\left(k_{v}\right)$ and $\left(\sigma_{v}\right)$ given above belong to a finite set of exceptional data up to twisting.

This theorem affirms the existence, at least for GL(2), of automorphic representations subject to local constrains which are more stringent than those previously considered in the literature. Clozel Clo86], building on a result of DeGeorge and Wallach [DW78, begins with a real semisimple Lie group $G$ and a discrete series representation $\delta$ of $G$ and then uses a trace formula to count the multiplicity of $\delta$ in $L^{2}(\Gamma \backslash G)$ as $\Gamma$ ranges through a tower of arithmetic subgroups of $G$ : This is akin to counting the number of modular forms of a given weight $k$ whose level is supported on a finite set of primes, though it may be deeply ramified at those primes. Chenevier [Che07] uses the PeterWeyl theorem to construct automorphic representations of a certain sort of unitary group (which is compact at the infinite places) which has prescribed ramification at a prime but arbitrary behavior at infinity; this representation is then used to construct number fields with given ramification. Our present investigation is limited to the group GL(2), but we control both the weight and the ramification at all finite places. In this sense our main theorem is similar to a theorem of Khare and Prasad [KP96], which shows the existence of classical cuspidal eigenforms of weight 2 for the principal congruence subgroup $\Gamma(p)$ which have a particular sort of ramification behavior at one prime $p$. In Section 4.3 we offer some very detailed information in the case of $K=\mathrm{Q}$; in particular, we compute the class of the space of cusp forms $S_{k}(\Gamma(N))$ in the Grothendieck group of $\mathrm{SL}(2, \mathbf{Z} / N \mathbf{Z})$, thus generalizing the classical dimension formulas in Shi71] and CO77.

To state our main theorem, we need to introduce some notation regarding "inertial types" for $G L(2)$ over a local or global field.

1.1. Local Inertial Types. Let $F$ be a finite extension of $\mathbf{Q}_{p}$, with ring of integers $\mathcal{O}_{F}$. Let $\mathcal{A}_{2}(F)$ be the set of isomorphism classes of complex-valued irreducible admissible representations of $\operatorname{GL}(2, F)$. By the local Langlands correspondence, there is a bijection $\pi \mapsto \sigma(\pi)$ mapping $\mathcal{A}_{2}(F)$ onto the set of isomorphism classes of two-dimensional Frobenius-semisimple Weil-Deligne representations of $F$ preserving $L$ functions and epsilon factors, see for instance [Kut80]. (See [Tat79] for the definition of Weil-Deligne representation and for the construction of its $L$-function and epsilon factor.)

Let $\mathcal{O}_{F}$ be the ring of integers of $F$, so that $\mathrm{GL}\left(2, \mathcal{O}_{F}\right)$ is a maximal compact open subgroup of $\mathrm{GL}(2, F)$. In [Hen02] it is shown that if $\pi \in \mathcal{A}_{2}(F)$, then $\left.\pi\right|_{\mathrm{GL}\left(2, \mathcal{O}_{F}\right)}$ contains an irreducible finite-dimensional 
subspace $\tau(\pi)$ of $\mathrm{GL}\left(2, \mathcal{O}_{F}\right)$ which characterizes the restriction of $\sigma(\pi)$ to the inertia group of $F$. We shall call $\tau(\pi)$ the inertial type of $\pi$. We will leave the precise definition of $\tau(\pi)$ for Section 2 , but we remark that when $\pi$ belongs to the unramified principal series, $\tau(\pi)$ is the trivial representation. Let Types $(F)$ be the set of isomorphism classes of representations of $\mathrm{GL}\left(2, \mathcal{O}_{F}\right)$ which arise as inertial types for members of $\mathcal{A}_{2}(F)$. For $\tau \in \operatorname{Types}(F)$ we define the quantity

$$
d(\tau)= \begin{cases}q-1, & \tau \text { is the type of a special representation, } \\ \operatorname{dim} \tau, & \text { otherwise; }\end{cases}
$$

here $q$ is the cardinality of the residue field of $\mathcal{O}_{F}$. (A special representation of $\mathrm{GL}(2, F)$ is a twist of the Steinberg representation of this group. Weil-Deligne representations corresponding to special representations are precisely those with nontrivial monodromy operator.)

Now suppose $F=\mathbf{R}$. When $k \geq 2$ and $w$ are two integers of the same parity, let $\mathcal{D}_{k, w}$ be the essentially discrete series representation of $\mathrm{GL}(2, \mathbf{R})$ as in 0.2 of $\mathrm{Car} 86$. Then the central character of $\mathcal{D}_{k, w}$ is $t \mapsto t^{-w}$. Let Types $(F)$ denote the set of all such representations $\mathcal{D}_{k, w}$. If $\pi$ is such a representation we simply define $\tau(\pi)=\pi$. We define the function $d$ on $\operatorname{Types}(F)$ by $d\left(\mathcal{D}_{k, w}\right)=k-1$.

In either case, suppose $\tau \in \operatorname{Types}(F)$. When $\chi$ is a (one-dimensional) character of $F^{*}$ we denote by $\tau \otimes \chi$ the representation $g \mapsto \chi(\operatorname{det} g) \tau(g)$; this also belongs to Types $(F)$.

1.2. Global Inertial Types. Now suppose $K$ is a totally real field of degree $n$. Let $S$ (resp., $S_{f}, S_{\infty}$ ) be the set of places (resp., finite places, infinite places) of $K$. To a cuspidal automorphic representation $\pi$ of $\mathrm{GL}(2)_{K}$ arising from a Hilbert modular form, we can associate the representation $\tau(\pi)=\otimes_{v \in S} \tau\left(\pi_{v}\right)$ of $\operatorname{GL}\left(2, \hat{\mathcal{O}}_{K} \times(K \otimes \mathbf{R})\right)$. Loosely speaking, $\tau(\pi)$ measures the ramification of $\pi$ at the finite places and records the components of $\pi$ at the infinite places. If the collection of infinite places is denoted $\left\{v_{1}, \ldots, v_{n}\right\}$ and if $\pi_{v_{i}} \cong \mathcal{D}_{k_{i}, w_{i}}$, then $w_{1}=\cdots=w_{n}$ and the integers $k_{i}$ and $w_{i}$ all have the same parity. Such a representation $\pi$ arises from a Hilbert modular form of weights $\left(k_{1}, \ldots, k_{d}\right)$, $c f$. Oht83.

We have that $\tau\left(\pi_{v}\right)$ is the trivial representation for all finite places not dividing the level of $\pi$. Furthermore, the central character $\chi$ of $\pi$ is an algebraic Hecke character of $\mathbf{A}_{K}^{*}$ whose restriction to $\mathcal{O}_{K, v}^{*}$ (resp., $K_{v}^{*}$ ) equals the central character of $\tau\left(\pi_{v}\right)$ for all $v \in S_{f}$ (resp., $v \in S_{\infty}$ ). In light of this we define the set Types $(K)$ of global inertial types to consist of the collections $\tau=\left(\tau_{v}\right)_{v \in S}$ satisfying the conditions: 
(1) For all but finitely many $v, \tau_{v}$ is the trivial representation.

(2) There exists an algebraic Hecke character of $\mathbf{A}_{K}$ whose component at each place $v$ agrees with the central character of $\tau_{v}$. (If one exists, then there are as many as the class number of $F$.)

We remark that condition (2) is equivalent to the condition that if $\chi_{v}$ is the central character of $\tau_{v}$, then

$$
\chi=\prod_{v} \chi_{v} \text { vanishes on } \mathcal{O}_{K}^{*} .
$$

Indeed, $\chi$ is a character of the subgroup $\prod_{v \in S_{f}} \mathcal{O}_{K_{v}}^{*} \times \prod_{v \in S_{\infty}} K_{v}^{*}$ of $\mathbf{A}_{K}^{*}$, and for $\chi$ to extend to an algebraic Hecke character it is necessary and sufficient that $\chi$ vanish on the intersection of this subgroup with the diagonally embedded group $K^{*} \subset \mathbf{A}_{K}^{*}$. This intersection is exactly the unit group $\mathcal{O}_{K}^{*}$.

If $\tau \in \operatorname{Types}(K)$ is a global inertial type, we shall write $\tau=\tau_{f} \otimes \tau_{\infty}$ to denote the decomposition of $\tau$ into its finite and infinite components. Note that $\tau_{f}$ is a finite-dimensional representation of the compact group $\operatorname{GL}\left(2, \hat{\mathcal{O}}_{F}\right)$ and $\tau_{\infty}$ is a representation of $\operatorname{GL}(2, K \otimes \mathbf{R})$. If we write $\tau_{v_{i}} \cong D_{k_{i}, w_{i}}$ for the infinite places $v_{i}$, then Eq. 1.1 and the Dirichlet Unit Theorem imply that $w_{1}=\cdots=w_{n}$.

Whenever $\pi$ is a cuspidal automorphic representation of $\mathrm{GL}(2)_{K}$ arising from a Hilbert modular form, $\tau(\pi)$ belongs to Types $(K)$. For any $\tau \in \operatorname{Types}(K)$, we define

$$
d(\tau)=\prod_{v} d\left(\tau_{v}\right)
$$

the product making sense because all but finitely many factors are 1 .

The notion of a global type $\tau$ being a twist of another type $\tau^{\prime}$ is evident: this shall mean that for each finite (resp. infinite) place $v$ there exist characters $\chi_{v}$ of $\mathcal{O}_{v}^{*}$ (resp. $K_{v}^{*}$ ) such that $\tau_{v}=\tau_{v}^{\prime} \otimes \chi_{v}$ for all places. (This can only be so if $\prod_{v} \chi_{v}^{2}$ vanishes on $\mathcal{O}_{K}^{*}$.)

1.3. Main Theorem. Let $S(\tau)$ denote the set of Hilbert modular forms $\pi$ for which $\tau(\pi)=\tau$. Our main theorem is an estimate for the cardinality of $S(\tau)$.

Theorem 1.1. We have

$$
\# S(\tau)=2^{1-n}\left|\zeta_{K}(-1)\right| h d(\tau)+O\left(2^{\nu(\tau)}\right),
$$

where

$$
\begin{aligned}
\zeta_{K}(s) & =\text { the Dedekind zeta function for } K \\
h & =\text { the class number of } K \\
\nu(\tau) & =\text { the number of finite places } v \text { for which } \operatorname{dim} \tau_{v}>1 .
\end{aligned}
$$


The constant in the "O" only depends on the field $K$.

Note that $\zeta_{K}(-1)$ is a nonzero (in fact rational) number. By comparing the quantity $d(\tau)$ with the error term $2^{\nu(\tau)}$ (see Section 2.1), we will deduce the following

Corollary 1.2. Up to twisting by one-dimensional characters, the set of global inertial types $\tau \in \operatorname{Types}(K)$ for which $S(\tau)=\emptyset$ is finite.

Stated rather loosely, this means that there always exists a Hilbert modular form with prescribed ramification data, so long as: the desired weight is large enough at one of the infinite places, OR the desired inertial representation at finite place $v$ is ramified deeply enough, OR enough primes are permitted to ramify. See Section 4.3 for an explicit account of the case $K=\mathbf{Q}$.

We have found it most convenient to divide the proof of Theorem 1.1 into two cases, depending on the parity of $n=[K: \mathbf{Q}]$. If $n$ is even, we work with the definite quaternion algebra $D$ ramified exactly at the infinite places. If $n$ is odd, we work with the quaternion algebra $D$ ramified at all but one of the infinite places. In each case, we wish to compute the multiplicity of a global type in a space of automorphic forms on a Shimura variety corresponding to $D$. The dimension of the Shimura variety will be 0 or 1 as $n$ is even or odd, respectively.

\section{TYPES FOR GL(2)}

2.1. Definition of Types. Let $F$ be a $p$-adic field with ring of integers $\mathcal{O}_{F}$, maximal ideal $\mathfrak{p}_{F}$, and residue field $\mathbf{F}_{q}$. In this section we gather the necessary definitions and facts concerning types for $\mathrm{GL}(2, F)$.

We must first define the association $\pi \mapsto \tau(\pi)$ attaching types to objects of $\mathcal{A}_{2}(F)$. We do this by cases as follows. It will simplify matters to assume in the following discussion that $\pi$ is of minimal conductor among its twists by characters of $F^{*}$. Types for $\pi$ not satisfying this condition can be defined via the relation $\tau(\pi \otimes \chi)=\left.\tau(\pi) \otimes \chi\right|_{\mathcal{O}_{F}^{*}}$.

(1) If $\pi$ belongs to the unramified principal series then $\tau(\pi)$ is the trivial representation of $\mathrm{GL}\left(2, \mathcal{O}_{F}\right)$.

(2) If $\pi$ is the principal series representation corresponding to a pair of characters $\chi_{1}, \chi_{2}$ of $F^{*}$ with $\chi_{1} \chi_{2}^{-1}$ ramified, twist $\pi$ so as to assume that $\chi_{2}$ is trivial. Let $\mathfrak{p}^{\mathfrak{c}}$ be the conductor of $\chi_{1}$. Then $\tau(\pi)$ is induced from the character $\left(\begin{array}{ll}a & b \\ c & d\end{array}\right) \mapsto \chi_{1}(a)$ of

$$
\Gamma_{0}\left(\mathfrak{p}^{\mathfrak{c}}\right):=\left\{\left(\begin{array}{ll}
a & b \\
c & d
\end{array}\right) \in \operatorname{GL}\left(2, \mathcal{O}_{F}\right) \mid c \equiv 0 \quad\left(\bmod \mathfrak{p}^{\mathfrak{c}}\right)\right\} .
$$


By Cas73, Prop. 1, $\tau(\pi)$ is an irreducible representation of $\operatorname{GL}\left(2, \mathcal{O}_{F}\right)$. We then have $d(\pi)=\operatorname{dim} \tau(\pi)=q^{c-1}(q+1)$.

(3) If $\pi=$ St is the Steinberg representation of $\operatorname{GL}(2, F)$, then $\tau(\pi)$ is pulled back from the unique irreducible $q$-dimensional representation $\mathrm{St}_{\mathrm{GL}\left(2, \mathcal{O}_{F}\right)}$ of $\mathrm{GL}\left(2, \mathbf{F}_{q}\right)$ contained in the permutation representation on $\mathbf{P}^{1}\left(\mathbf{F}_{q}\right)$. In this case we have defined $d(\tau(\pi))=q-1$

(4) If $\pi$ is supercuspidal, then it is induced from a finite-dimensional irreducible representation $\lambda$ of a compact-mod-center subgroup $J \subset \mathrm{GL}(2, F)$. These were constructed in [Kut78a] and Kut78b; we review the construction in Section 2.2 , By replacing $J$ with a conjugate we may assume that the maximal compact subgroup $J^{0}$ of $J$ lies in $\operatorname{GL}\left(2, \mathcal{O}_{F}\right)$; then $\tau(\pi)=\left.\operatorname{Ind}_{J^{0}}^{\mathrm{GL}\left(2, \mathcal{O}_{F}\right)} \lambda\right|_{J^{0}}$ is irreducible; see [Hen02], A.3.1. When $\sigma(\pi)$ is induced from a character $\theta$ of a quadratic extension $F / F$ of conductor $c \geq 1$, we will see in the next section that the dimension of $\tau(\bar{\pi})$ is given by

$$
\operatorname{dim} \tau(\pi)= \begin{cases}q^{c-1}(q-1), & F / F \text { unramified } \\ q^{c-2}\left(q^{2}-1\right), & F / F \text { ramified }\end{cases}
$$

We shall call a type $\tau(\pi)$ principal series (resp., special, supercuspidal) when $\pi$ is principal series (resp., special, supercuspidal). From [Hen02], A.1.5 we deduce the theorem:

Theorem 2.1. Let $\pi, \pi^{\prime} \in \mathcal{A}_{2}(F)$. The following are equivalent:

(1) $\left.\pi^{\prime}\right|_{\mathrm{GL}\left(2, \mathcal{O}_{F}\right)}$ contains $\tau(\pi)$.

(2) $\left.\left.\sigma(\pi)\right|_{I_{F}} \cong \sigma(\pi)\right|_{I_{F}} ^{\prime}$ or $\pi=\chi \otimes$ St and $\pi^{\prime}$ is the principal series representation attached to two unequal characters of $F^{*}$ whose restriction to $\mathcal{O}_{F}^{*}$ agrees with $\left.\chi\right|_{\mathcal{O}_{F}^{*}}$.

Therefore if $\pi, \pi^{\prime} \in \mathcal{A}_{2}(F)$ and $\pi^{\prime}$ contains $\tau(\pi)$, then $\tau\left(\pi^{\prime}\right)=\tau(\pi)$, unless we are in the case of the "or" clause above, in which case $\tau(\pi)$ is a one-dimensional character $\chi$ and $\tau\left(\pi^{\prime}\right)=\chi \otimes \operatorname{St}_{\mathrm{GL}\left(2, \mathcal{O}_{F}\right)}$.

A word on the dimensions of types is in order. Suppose $\tau$ runs through a sequence of irreducible representations of $\operatorname{GL}\left(2, \mathcal{O}_{F}\right)$. If we assume that no member of this sequence is the twist of any other by a one-dimensional character, then we must have $\operatorname{dim} \tau \rightarrow \infty$. Of course this implies $d(\tau) \rightarrow \infty$ as well. Furthermore, an irreducible representation of $\mathrm{GL}\left(2, \mathcal{O}_{F}\right)$ of least dimension other than 1 is one that is inflated from a cuspidal representation of $\operatorname{GL}\left(2, \mathbf{F}_{q}\right)$, and this has dimension $q-1$. Therefore we have the lower bound $d(\tau) \geq q-1$ whenever $\tau$ is not one-dimensional. 
We shall now deduce Cor. 1.2 from Thm. 1.1. Let $K$ be a totally real field and suppose that $\tau$ runs through a sequence of global inertial types of $K$, no two of which are twists of each other. To prove the corollary it will suffice to show that the sequence $d(\tau) / 2^{\nu(\tau)}$ tends to infinity. Assume instead that it is bounded. By the previous paragraph we have the lower bound

$$
d(\tau) / 2^{\nu(\tau)} \geq \prod_{v} \frac{q_{v}-1}{2}
$$

where the product runs over the finite places $v$ for which $\tau_{v}$ is not onedimensional. Since the left hand side of this is bounded, the global types $\tau$ can only have higher-dimensional components at a finite set $S_{0}$ of finite places. This implies that $\nu(\tau)$ is bounded from above; by the hypothesis that $d(\tau) / 2^{\nu(\tau)}$ is bounded we must have that $d(\tau)$ is bounded as well. We claim there must exist a place $v_{0} \in S_{0}$ for which $\tau_{v_{0}}$ assumes infinitely many distinct values up to twisting. The alternative is that for each place $v$ in $S_{0}$, the sequence $\tau_{v}$ comprises only finitely many types along with their twists; by the pigeonhole principle, this would imply that the sequence $\tau$ contains two types which are twists of one another, contradicting the hypothesis. But then by the previous paragraph $d\left(\tau_{v_{0}}\right) \rightarrow \infty$, contradicting the boundedness of $d(\tau)$. The corollary is proved.

2.2. Trace bounds for types. Define the level of a type $\tau$ to be the least integer $\ell$ for which $\tau$ factors through $\operatorname{GL}\left(2, \mathcal{O}_{F} / \mathfrak{p}^{\ell}\right)$. We shall say that the essential level of $\tau$ is the least level of all the twists of $\ell$.

We need a lemma concerning the non-archimedean local types.

Lemma 2.2. Let $g \in \mathrm{GL}\left(2, \mathcal{O}_{F}\right)$ be a matrix whose characteristic polynomial has discriminant in $\mathcal{O}_{F}^{*}$. Then for all $\tau \in \operatorname{Types}(F)$, we have

$$
\begin{cases}|\operatorname{Tr}(\tau(g))|=1, & \tau \text { one-dimensional or special }, \\ |\operatorname{Tr}(\tau(g))| \leq 2, & \text { all other cases. }\end{cases}
$$

Furthermore, if we relax the hypothesis on $g$ and merely assume that $g$ has distinct eigenvalues in $\bar{F}^{*}$, then $|\operatorname{Tr} \tau(g)|$ is bounded as $\tau$ ranges through Types $(F)$.

Proof. We proceed by taking cases with respect to the structure of $\tau$. If $\tau$ is one-dimensional, the inequality of the lemma is obvious. If $\tau$ is special, then up to twisting it is the inflation of the Steinberg representation $\mathrm{St}_{\mathrm{GL}\left(2, \mathbf{F}_{q}\right)}$. If the characteristic polynomial of $g \in \mathrm{GL}\left(2, \mathcal{O}_{F}\right)$ has unit discriminant then its reduction $\bar{g} \in \mathrm{GL}\left(2, \mathbf{F}_{q}\right)$ has distinct 
eigenvalues. From the formula

$$
\operatorname{St}_{\mathrm{GL}\left(2, \mathbf{F}_{q}\right)}=\left[\operatorname{Ind}_{B}^{\mathrm{GL}\left(2, \mathbf{F}_{q}\right)} 1\right]-[1], B=\left\{\left(\begin{array}{ll}
a & b \\
0 & d
\end{array}\right)\right\},
$$

we see easily that $\operatorname{Tr}\left(\operatorname{St}_{\mathrm{GL}\left(2, \mathbf{F}_{q}\right)}(\bar{g})\right)$ equals 1 if $\bar{g}$ has eigenvalues in $\mathbf{F}_{q}^{*}$ and -1 otherwise, thus establishing Eq. 2.1. Of course we have $\mid \operatorname{Tr} \tau(g)) \mid \leq \operatorname{dim} \tau=q$ no matter what $g$ is.

We now give a more explicit description of $\tau$ in the remaining cases. In each of these cases $\tau$ is induced from a "small" representation of a subgroup of $\mathrm{GL}\left(2, \mathcal{O}_{F}\right)$. Since we are interested in the trace of $\tau$, the following form of Mackey's theorem will be useful:

$$
\operatorname{Tr}\left(\operatorname{Ind}_{H}^{G} \eta\right)(g)=\sum_{[x] \in(G / H)^{g}} \operatorname{Tr} \eta\left(x^{-1} g x\right),
$$

where the sum ranges over cosets $[x]=x H \in G / H$ which are fixed under the left action of $g$. We will be applying this equation to various finite-index subgroups $J^{0} \subset \mathrm{GL}\left(2, \mathcal{O}_{F}\right)$.

Suppose $\tau$ is the type of a ramified principal series representation. By replacing $\tau$ with a twist we may assume, as in the previous subsection, that $\tau$ is induced from the character $\left(\begin{array}{ll}a & b \\ c & d\end{array}\right) \mapsto \chi(a)$ of $\Gamma_{0}\left(\mathfrak{p}^{\mathfrak{c}}\right)$. Note that we can identify the quotient $\operatorname{GL}\left(2, \mathcal{O}_{F}\right) / \Gamma_{0}\left(\mathfrak{p}^{\mathfrak{c}}\right)$ with the projective line $\mathbf{P}^{1}\left(\mathcal{O}_{F} / \mathfrak{p}^{\mathfrak{c}}\right)$, together with its natural action of $\operatorname{GL}\left(2, \mathcal{O}_{F}\right)$. If $\bar{g}$ has distinct eigenvalues then $g$ only has zero or two fixed points on $\mathbf{P}^{1}\left(\mathcal{O}_{F} / \mathfrak{p}^{\mathfrak{c}}\right)$ and therefore $|\operatorname{Tr} \tau(g)| \leq 2$ in this case. If we lift this assumption and merely assume that $g$ has distinct eigenvalues in $\bar{F}^{*}$, then $g$ may have more than two fixed points on $\mathbf{P}^{1}\left(\mathcal{O}_{F} / \mathfrak{p}^{\mathfrak{c}}\right)$. However, we claim that asc $\rightarrow \infty$ then this number of fixed points remains bounded. To establish this, we may pass from $F$ to an extension $F$ containing the eigenvalues of $g$ and then show that $g$ has a bounded number of fixed points on $\mathbf{P}^{1}\left(\mathcal{O}_{F} / \mathfrak{p}_{F}^{\mathfrak{c}}\right)$ as $c \rightarrow \infty$. Working over $F$, we have up to conjugacy $g=\left(\begin{array}{cc}\alpha & \\ & \beta\end{array}\right)$, with $\alpha, \beta \in \mathcal{O}_{F}^{*}$; let $m$ be the valuation in $F$ of $\alpha-\beta$. Then for $\mathfrak{c} \geq m$, the fixed points of $g$ on $\mathbf{P}^{1}\left(\mathcal{O}_{F} / \mathfrak{p}_{F}^{\mathfrak{c}}\right)$ are exactly the points $[x: 1]$ and $[1: y]$, where $x$ and $y$ range through those elements of $\mathcal{O}_{F} / \mathfrak{p}^{\mathfrak{c}}$ of valuation at least $c-m$. Thus $g$ has at most $2\left(\# \mathcal{O}_{F} / \mathfrak{p}_{F}\right)^{m}$ fixed points which are $F$-rational, and this number is bounded as $\mathfrak{c} \rightarrow \infty$, proving the claim. Therefore $|\operatorname{Tr} \tau(g)|$ is bounded as $\tau$ ranges through all types of ramified principal series representations.

Now suppose $\tau$ is the type of a minimal supercuspidal representation $\pi$. To prove the required inequality, we need to give a more detailed 
description of $\tau$. Here we follow Hen02, A.3.2-A.3.8, wherein an exhaustive list of the supercuspidal types is given. Let $k=\mathcal{O}_{F} / \mathfrak{p}_{F}$, let $\varpi_{F}$ be a uniformizer of $F$, and let $\psi$ be an additive character of $F$ which vanishes on $\mathfrak{p}_{F}$ but not on $\mathcal{O}_{F}$. There are three cases to consider:

(1) $\pi$ has conductor $\mathfrak{p}^{2}$.In this case $\tau$ is inflated from an irreducible cuspidal representation of $\mathrm{GL}_{2}(k)$. These in turn are in correspondence with certain characters $\theta$ of the multiplicative group of a quadratic extension $k_{2} / k$. If $\theta$ is a character of $k_{2}^{*}$ unequal to its $k$-conjugate, then there is a unique $(q-1)$-dimensional representation $\tau_{\theta}$ of $\mathrm{GL}(2, k)$ satisfying

$\operatorname{Tr} \tau_{\theta}(\bar{g})= \begin{cases}-\left(\theta\left(\alpha_{1}\right)+\theta\left(\alpha_{2}\right)\right), & \bar{g} \text { has eigenvalues } \alpha_{1}, \alpha_{2} \in k_{2}^{*} \backslash k^{*} \\ 0, & \bar{g} \text { has distinct eigenvalues in } k^{*}\end{cases}$

Then the type $\tau$ is inflated from one of the representations $\tau_{\theta}$. The desired inequality $|\operatorname{Tr} \tau(g)| \leq 2$ is obvious from the above description so long as $\bar{g}$ has distinct eigenvalues.

(2) $\pi$ has conductor $\mathfrak{p}_{F}^{\mathfrak{c}}, \mathfrak{c} \geq 4$ even. Let $E / F$ be the unique unramified quadratic extension field, and let $n=\mathfrak{c} / 2$. Let $b \in E^{*}$ be of the form $\varpi^{-n} u$, where $u \in \mathcal{O}_{E}^{*}$ has residue class in $\mathcal{O}_{E} / \mathfrak{p}_{E}$ which generates that field over $k$. Let $\theta$ be a character of $E^{*}$ for which $\theta(1+x)=\psi \circ \operatorname{Tr}_{E / F}(b x)$ for $b \in \mathfrak{p}_{E}^{\lfloor(n+1) / 2\rfloor}$.

Choose an embedding $E \hookrightarrow M_{2}(F)$ so that $M_{2}\left(\mathcal{O}_{F}\right) \cap E=$ $\mathcal{O}_{E}$. Define the subgroup $J^{0} \subset \mathrm{GL}\left(2, \mathcal{O}_{F}\right)$ by

$$
J^{0}=\mathcal{O}_{E}^{*}\left(1+\mathfrak{p}_{F}^{\lfloor(n+1) / 2\rfloor} M_{2}\left(\mathcal{O}_{F}\right)\right) .
$$

Then $\tau=\operatorname{Ind}_{J^{0}}^{\mathrm{GL}\left(2, \mathcal{O}_{F}\right)} \eta$ for a representation $\eta$ of $J^{0}$ which we now describe. If $n$ is odd then $\eta$ is a character of $J^{0}$ defined by the conditions $\left.\eta\right|_{\mathcal{O}_{E}^{*}}=\left.\theta\right|_{\mathcal{O}_{E}^{*}}$ and $\theta(1+x)=\psi \circ \operatorname{Tr}(b x)$ for $x \in \mathfrak{p}_{F}^{(n+1) / 2} M_{2}\left(\mathcal{O}_{F}\right)$. If $n$ is even then $\eta$ is the unique irreducible representation of $J^{0}$ of dimension $q$ satisfying $\operatorname{Tr} \eta(\alpha(1+x))=$ $-\theta(\alpha)$ whenever $x \in \mathfrak{p}_{F}^{(n+1) / 2}$ and $\alpha \in \mathcal{O}_{E}^{*}$ is such that the image of $\alpha$ in $\mathcal{O}_{E} / \mathfrak{p}_{E}$ does not lie in $k$. We remark that representations $\pi$ of $\operatorname{GL}(2, F)$ containing the type $\tau$ have conductor $\mathfrak{p}_{F}^{2 n}=\mathfrak{p}_{F}^{\mathfrak{c}}$.

To apply Mackey's theorem we need to consider the coset space $\operatorname{GL}\left(2, \mathcal{O}_{F}\right) / J^{0}$ together with its left $\mathrm{GL}\left(2, \mathcal{O}_{F}\right)$-action. If we let

$$
\mathcal{H}_{n}=\left\{\alpha \in\left(\mathcal{O}_{E} / \mathfrak{p}_{E}^{\lfloor(n+1) / 2\rfloor}\right)^{*} \mid \alpha \bmod \mathfrak{p}_{E} \notin k\right\},
$$

then there is a natural left action $(g, b) \mapsto g \cdot b$ of $\mathrm{GL}\left(2, \mathcal{O}_{F}\right)$ on $\mathcal{H}_{c}$ via fractional linear transformations. If $b \in \mathcal{H}_{n}$ is a 
fixed point of $\mathcal{O}_{E}^{*}$ then $g \mapsto g \cdot b$ gives a $\mathrm{GL}\left(2, \mathcal{O}_{F}\right)$-equivariant bijection

$$
\mathrm{GL}\left(2, \mathcal{O}_{F}\right) / J^{0} \cong \mathcal{H}_{n}
$$

much as the coset space $\operatorname{GL}(2, \mathbf{R}) / O(2, \mathbf{R})$ is identified with (two copies of) the upper half plane. If $g \in \mathrm{GL}\left(2, \mathcal{O}_{F}\right)$ is such that $\bar{g}$ has distinct eigenvalues, then $g$ has at most two fixed points in $\mathcal{H}_{n}$ and we find $|\operatorname{Tr} \tau(g)| \leq 2$. (In fact if $g$ has eigenvalues $\alpha, \beta$ lying in $\mathcal{O}_{F^{\prime}}$ then $\operatorname{Tr} \tau(g)=(-1)^{n-1}(\theta(\alpha)+\theta(\beta))$.) On the other hand if $g$ is merely assumed to have distinct eigenvalues then $g$ may have more than two fixed points on $\mathcal{H}_{n}$. However, the number of fixed points is bounded as $n \rightarrow \infty$, by the same argument given in the previous paragraph. Therefore $|\operatorname{Tr} \tau(g)|$ is bounded as $\tau$ runs through types of supercuspidal representations of this sort.

(3) $\pi$ has conductor $\mathfrak{p}_{F}^{\mathfrak{c}}, \mathfrak{c} \geq 3$ odd. Let $E / F$ be a ramified quadratic extension, and let $n=\mathfrak{c}-2$. Let $b \in E^{*}$ have valuation $-n$. Let $\theta$ be a character of $E^{*}$ satisfying $\theta(1+x)=\psi \circ \operatorname{Tr}_{E / F}(b x)$ whenever $x \in \mathfrak{p}_{E}^{(n+1) / 2}$.

Let $\mathfrak{A} \subset M_{2}\left(\mathcal{O}_{F}\right)$ be the algebra

$$
\mathfrak{A}=\left\{\left(\begin{array}{ll}
a & b \\
c & d
\end{array}\right) \mid c \in \mathfrak{p}_{F}\right\},
$$

and choose an embedding $E \hookrightarrow M_{2}(F)$ in such a way that $\mathfrak{A} \cap E=\mathcal{O}_{E}^{*}$. Let $P_{\mathfrak{A}} \subset \mathfrak{A}$ be the double-sided ideal of matrices $\left(\begin{array}{ll}a & b \\ c & d\end{array}\right)$ with $a, c, d \in \mathfrak{p}_{F}$. Our subgroup $J^{0}$ is then

$$
J^{0}=\mathcal{O}_{E}^{*}\left(1+P_{\mathfrak{A}}^{(n+1) / 2}\right)
$$

and $\eta$ is the character $\alpha(1+x) \mapsto \theta(\alpha) \psi(\operatorname{Tr}(b x))$ for $\alpha \in \mathcal{O}_{E}^{*}$, $x \in P_{\mathfrak{A}}^{(n+1) / 2}$. Then $\tau=\operatorname{Ind}_{J^{0}}^{\mathrm{GL}\left(2, \mathcal{O}_{F}\right)} \eta$ is a type contained in supercuspidal representations $\pi$ of $\mathrm{GL}(2, F)$ of conductor $n+2$.

Now suppose $g \in \mathrm{GL}\left(2, \mathcal{O}_{F}\right)$ is such that $\bar{g}$ has distinct eigenvalues. If $\bar{g}$ has irreducible characteristic polynomial, then no conjugate of $g$ can possibly lie in $\mathcal{A}^{*}$, let alone in $J^{0}$, so that $\operatorname{Tr} \tau(g)=0$. The alternative is that up to conjugacy $g \in \mathfrak{A}^{*}$ equals the diagonal matrix with eigenvalues $\alpha, \beta \in \mathcal{O}_{F}^{*}$ whose residue classes are unequal. Let $\mathcal{H}_{n}$ be the quotient of the set $\mathfrak{p}^{-1} \backslash \mathcal{O}_{E}$ by the group $1+\mathfrak{p}_{E}^{n}$. The analogue of Eq. 2.3 is the $\mathfrak{A}^{*}$ equivariant bijection $\mathfrak{A}^{*} / J^{0} \cong \mathcal{H}_{n}$. Since $g$ has no fixed points on $\mathcal{H}_{n}$ we have $\operatorname{Tr} \tau(g)=0$ as well. 
Now assume only that $g$ has distinct eigenvalues. Let $\lambda=$ $\operatorname{Ind}_{J^{0}}^{\mathfrak{A}^{*}} \eta$. Then by Mackeys Theorem $\operatorname{Tr} \tau(g)$ is a sum over at most \# GL $\left(2, \mathcal{O}_{F}\right) / \mathfrak{A}^{*}=q+1$ terms of the trace $\operatorname{Tr} \lambda$ evaluated on conjugates of $g$. The same argument from the previous paragraph shows that an element $h \in \mathfrak{A}^{*}$ with distinct eigenvalues has a bounded number of fixed points on $\mathcal{H}_{n}$ as $n \rightarrow \infty$. Thus $|\operatorname{Tr} \tau(g)|$ is bounded as $\tau$ runs through types of supercuspidal representations of this sort as well.

Lemma 2.2 has a global consequence which we will need in the sequel. Let $K$ be a totally real field and suppose $B / K$ is a quaternion algebra (possibly $M_{2}(K)$ ) which is split at all finite places. Suppose $\mathcal{O}_{B} \subset$ $B$ is a maximal order. Then for all finite places $v$ we may identify $\mathcal{O}_{B} \otimes_{\mathcal{O}_{F}} \mathcal{O}_{F_{v}}$ with $\operatorname{GL}\left(2, \mathcal{O}_{F_{v}}\right)$. Let $g_{v}$ be the image of $g \otimes 1$ under this isomorphism. If $\tau=\tau_{f} \otimes \tau_{\infty} \in \operatorname{Types}(K)$, let $\tau_{f}$ be the representation of $\mathcal{O}_{B}^{*}$ defined by $g \mapsto \prod_{v \text { finite }} \tau_{v}\left(g_{v}\right)$.

Lemma 2.3. Let $g \in \mathcal{O}_{B}$ be an element whose (reduced) characteristic polynomial has distinct eigenvalues in $\bar{K}^{*}$. There is a constant $C$ depending only on $g$ (and of course $K$ ) such that for all types $\tau=\tau_{f} \otimes \tau_{\infty} \in \operatorname{Types}(K),\left|\operatorname{Tr} \tau_{f}(g)\right| \leq C 2^{\nu(\tau)-n_{s p}(\tau)}$, where $n_{s p}(\tau)$ is the number of finite places of $K$ at which $\tau$ is special.

Proof. Indeed, if such a $g$ is given then only finitely many finite places of $K$ will divide the discriminant of the characteristic polynomial of $g$. Let $S_{g}$ be the set of such places. For each $v \in S_{g}$, the preceding lemma shows that there exists a bound $C_{v}$ so that for all $\tau_{v} \in \operatorname{Types}\left(K_{v}\right)$ we have $\left|\operatorname{Tr} \tau_{v}(g)\right| \leq C_{v}$. Let $C=\prod_{v \in S_{g}} C_{v}$. For every finite place $v \notin S_{g}$, Lemma 2.1 shows that $\left|\operatorname{Tr} \tau_{v}\left(g_{v}\right)\right| \leq 1$ if $\tau_{v}$ is special or onedimensional and $\left|\operatorname{Tr} \tau_{v}\left(g_{v}\right)\right| \leq 2$ otherwise. Therefore if $\tau_{f}$ is the finite part of a global type, we have the inequality

$$
\left|\operatorname{Tr} \tau_{f}(g)\right|=\prod_{v \text { finite }}\left|\tau_{v}\left(g_{v}\right)\right| \leq\left(\prod_{v \in S_{g}} C_{v}\right) 2^{\nu(\tau)-n_{\mathrm{sp}}(\tau)}
$$

as required.

\section{Proof of Theorem 1.1 in the case of $[K: \mathbf{Q}]$ Even}

Assume that $n=[K: \mathbf{Q}]$ is even. Let $S_{f}$ and $S_{\infty}$ denote the finite and infinite places of $K$, respectively. Let $\mathbf{A}$ and $\mathbf{A}_{f}$ denote the adeles and finite adeles of $K$. Finally, let $D / K$ be the quaternion 
algebra ramified exactly at $S_{\infty}$, and let $G=D^{*}$ be the inner form of GL(2) corresponding to $D$. The Jacquet-Langlands correspondence JL : $\pi^{\prime} \mapsto \pi$ puts automorphic representations of $G(\mathbf{A})$ in bijection with those automorphic representations of $\mathrm{GL}(2, \mathbf{A})$ which are discrete series at the infinite places, see [JL70] We will also use the symbol JL to mean the local Jacquet-Langlands correspondence between representations of $D_{v}$ and those of $\mathrm{GL}\left(2, K_{v}\right)$ for any particular $v$.

Now suppose $\tau \in \operatorname{Types}(K)$ is a global type for $K$. Choose a maximal order $\mathcal{O}$ of $D$. For each finite place $v \in S_{0}$ there exists an isomorphism $\mathcal{O}_{v} \cong G L\left(2, \mathcal{O}_{K_{v}}\right)$, unique up to conjugacy. Let $\tau_{v}^{\prime}$ be the pull-back of $\tau_{v}$ through any such isomorphism; this is well-defined up to isomorphism. Let $\tau_{f}^{\prime}=\bigotimes_{v \in S_{f}} \tau_{v}^{\prime}$; this is a finite-dimensional irreducible representation of $\hat{\mathcal{O}}^{*}$. Let also $\tau_{\infty}^{\prime}=\bigotimes_{v \in S_{\infty}} \mathrm{JL}^{-1}\left(\tau_{v}\right)$; this is a finite-dimensional representation of $G(K \otimes \mathbf{R})=\prod_{v \in S_{\infty}} G\left(K_{v}\right)$. Finally, let $\tau^{\prime}=\tau_{f}^{\prime} \otimes \tau_{\infty}^{\prime}$, this is a representation of $G\left(\hat{\mathcal{O}}_{K} \times(K \otimes \mathbf{R})\right)$. We record the relationship

$$
d(\tau)=\operatorname{dim} \tau^{\prime} \prod_{v \in S_{\mathrm{sp}}}\left(1-\frac{1}{q_{v}}\right),
$$

where $S_{\mathrm{sp}}$ is the set of places at which $\tau$ is special and $q_{v}$ is the cardinality of the residue field of a finite place $v$.

Because multiplicity one holds for $\mathrm{GL}(2)$ and for $G$, we see that counting the automorphic representations of $G L\left(2, \mathbf{A}_{K}\right)$ whose restriction to $\mathrm{GL}\left(2, \hat{\mathcal{O}}_{K} \times(K \otimes \mathbf{R})\right)$ contains $\tau$ is the same as counting the automorphic representations of $G\left(\mathbf{A}_{K}\right)$ whose restriction to $G\left(\hat{\mathcal{O}}_{K} \times\right.$ $(K \otimes \mathbf{R})$ ) contains $\tau^{\prime}$. Write $\mu(\tau)$ for the number of automorphic representations. It is not necessarily the case that $\mu(\tau)$ is the cardinality of $S(\tau)$, the set of automorphic representations of type $\tau$, due to the possibility of special components. We will compute $\# S(\tau)$ in terms of $\mu(\tau)$ at the end of the section.

To compute $\mu(\tau)$, we first realize it as the multiplicity of $\tau_{f}^{\prime}$ inside a space of automorphic forms of "weight $\tau_{\infty}^{\prime}$ ", namely

$$
M\left(\tau_{\infty}^{\prime}\right)=\mathcal{C}_{G(K \otimes \mathbf{R})}\left(G(K) \backslash G(\mathbf{A}), \tau_{\infty}^{\prime}\right),
$$

the space of functions $f$ on $G(\mathbf{A})$ taking values in the vector space underlying $\tau_{\infty}^{\prime}$ which are left- $G(K)$-invariant and which satisfy $f\left(x g_{\mathbf{R}}^{-1}\right)=$ $\tau_{\infty}^{\prime}\left(g_{\mathbf{R}}\right) f(x)$ for $x \in G(\mathbf{A}), g_{\mathbf{R}} \in G(K \otimes \mathbf{R})$. This space has a left action of $G\left(\mathbf{A}_{f}\right)$ via $(g f)(x)=f(x g)$; the automorphic representations of $G$ which are $\tau_{\infty}^{\prime}$ at the infinite places are exactly the irreducible $G\left(\mathbf{A}_{f}\right)$ stable subrepresentations of $M\left(\tau_{\infty}^{\prime}\right)$. Because an element of $M\left(\tau_{\infty}^{\prime}\right)$ is 
determined by its restriction to $G\left(\mathbf{A}_{f}\right)$, we may rewrite $\mathrm{Eq}(3.2)$ as

$$
M\left(\tau_{\infty}^{\prime}\right)=\mathcal{C}_{G(K)}\left(G\left(\mathbf{A}_{f}\right), \tau_{\infty}^{\prime}\right) ;
$$

that is, the space of functions $f$ on $G\left(\mathbf{A}_{f}\right)$ with values in $V\left(\tau_{\infty}^{\prime}\right)$ satisfying $f(k g)=\tau_{\infty}^{\prime}(k) f(g)$ for $k \in G(K)$ and $g \in G\left(\mathbf{A}_{f}\right)$.

Let $T$ be a set of double coset representatives for the finite set $G(K) \backslash G\left(\mathbf{A}_{f}\right) / \hat{\mathcal{O}}^{*}$. Then $T$ is finite by |Vig80, Theoreme 5.4. For each $t \in T$, there is a corresponding maximal order $\mathcal{O}_{t}=t \hat{\mathcal{O}} t^{-1} \cap D$. Then the right-hand side of Eq (3.3) decomposes into a sum of $\hat{\mathcal{O}}^{*}$-stable spaces indexed by $T$ :

$$
M\left(\tau_{\infty}^{\prime}\right)=\bigoplus_{t \in T} \mathcal{C}_{G(K)}\left(G(K) t \hat{\mathcal{O}}^{*}, \tau_{\infty}^{\prime}\right)
$$

with some thought each summand on the right is seen to be isomorphic as a left $\hat{\mathcal{O}}^{*}$-module to $\operatorname{Ind}_{\mathcal{O}_{t}^{*}}^{\hat{\mathcal{O}}^{*}}\left(\tau_{\infty}^{\prime}\right)^{\vee}$, where $\mathcal{O}_{t}^{*}$ is to be regarded as a subgroup of $\hat{\mathcal{O}}^{*}$ via conjugation by $t$, and $\left(\tau_{\infty}^{\prime}\right)^{\vee}$ is the contragradient of $\tau_{\infty}^{\prime}$.

By Frobenius reciprocity, the desired multiplicity $\mu(\tau)$ is therefore a sum of terms

$$
\mu(\tau)=\sum_{t \in T} \mu_{t},
$$

where $\mu_{t}$ is the inner product of the $\mathcal{O}_{t}^{*}$-modules $\left.\tau_{f}^{\prime}\right|_{\mathcal{O}_{t}^{*}}$ and $\left.\left(\tau_{\infty}^{\prime}\right)^{\vee}\right|_{\mathcal{O}_{t}^{*}}$. That is, $\mu_{t}$ is the multiplicity of the trivial character in the restriction of $\tau^{\prime}=\tau_{f}^{\prime} \otimes \tau_{\infty}^{\prime}$ to $\mathcal{O}_{t}^{*}$. By Eq. 1.1, $\tau$ is trivial on $\mathcal{O}_{K}^{*}$; it therefore factors through the finite group $W_{t}=\mathcal{O}_{t}^{*} / \mathcal{O}_{K}^{*}$. Let $e_{t}$ be the order of $W_{t}$. Then

$$
\mu_{t}=\frac{1}{e_{t}} \sum_{\omega \in W_{t}} \operatorname{Tr} \tau^{\prime}(\omega)
$$

We claim that the term with $\omega=1$ dominates the sum in Eq. (3.4). Indeed, suppose $\omega \in \mathcal{O}_{t}^{*}$ is outside of $\mathcal{O}_{K}^{*}$. Since $\omega^{e_{t}}$ belongs to the center of $G(K)$ but $\omega$ itself does not, $\omega$ is semisimple. Therefore by Lemma 2.3.

$$
\left|\tau_{f}(\omega)\right| \leq C_{1} 2^{\nu(\tau)-n_{\mathrm{sp}(\tau)}}
$$

for a constant $C_{1}$. A priori this $C_{1}$ depends on $\omega$, but since there are only finitely many $\omega$ under consideration, we may take $C_{1}$ to depend only on $K$.

We now turn to the infinite places. For each infinite place $v$, let $\iota_{v}: K_{v} \cong \mathbf{R}$ be the corresponding isomorphism. We have that $G\left(K_{v}\right)$ is isomorphic to the group of quaternions $\alpha+\beta j$, with $\alpha, \beta$ complex 
numbers which are not both zero. Let $\rho_{v}: G\left(K_{v}\right) \rightarrow \mathrm{GL}(2, \mathbf{C})$ be the representation

$$
\alpha+\beta j \mapsto\left(\begin{array}{cc}
\alpha & \beta \\
-\bar{\beta} & \bar{\alpha}
\end{array}\right)
$$

so that the central character of $\rho_{v}$ is $\iota_{v}$. Suppose that $\tau_{v}$ is the discrete series representation $\mathcal{D}_{k_{v}, w}$ of $\operatorname{GL}(2, \mathbf{R})$. (Note that the integer $w$ will not depend on the place $v$.) Then

$$
\tau_{v}=\left(\iota_{v} \circ \mathrm{N}_{D_{v} / K_{v}}\right)^{1-\frac{k+w}{2}} \otimes \operatorname{Sym}^{k_{v}-2} \rho_{v},
$$

where $\mathrm{N}_{D_{v} / K_{v}}$ is the reduced norm from $D_{v}$ to $K_{v}$; see Car86], 0.10 .

Now suppose that $\omega$ is an element of $\mathcal{O}_{t}^{*} \backslash \mathcal{O}_{K}^{*}$. Let $\omega_{v}$ be the image of $\omega$ under $\rho_{v}$. Since $\omega_{v}^{e_{t}}$ is a scalar but $\omega_{v}$ is not, the eigenvalues of $\omega_{v}$ are $\zeta \alpha$ and $\zeta^{-1} \alpha$ for $\zeta \neq \pm 1$ satisfying $\zeta^{e_{t}}=1$ and $\alpha^{2}=\operatorname{det} w_{v}=$ $\iota\left(\mathrm{N}_{D_{v} / K_{v}} \omega\right)$. The trace of $\operatorname{Sym}^{k-2} \tau_{v}^{\prime}(\omega)$ is then $\left(\zeta^{k}+\zeta^{k-2}+\cdots+\right.$ $\left.\zeta^{-k}\right) \iota_{v}\left(\mathrm{~N}_{D_{v} / K_{v}} \omega\right)(k-2) / 2$. The sum of roots of unity is bounded by a constant $C_{2}$ which depends only on $e_{t}$, and since $t$ runs over a finite set, this constant may be taken to depend only on $K$. Therefore for each $v \mid \infty$ we have

$$
\left|\operatorname{Tr} \tau_{v}^{\prime}(\omega)\right| \leq C_{2} \iota_{v}\left(\mathrm{~N}_{D_{v} / K_{v}} \omega\right)^{-w / 2}
$$

Multiplying Eq. 3.7] for $v \in S_{\infty}$ gives

$$
\left|\operatorname{Tr} \tau_{\infty}^{\prime}(\omega)\right| \leq C_{2}^{n}\left(\prod_{v \mid \infty} \mathrm{N}_{D_{v} / K_{v}} \omega\right)^{-w / 2}=C_{2}^{n},
$$

where in the last step we have used the product formula together with the fact that the reduced norm of $\omega$ belongs to $\mathcal{O}_{K}^{*}$. Putting together Eqs. 3.5 and 3.8 we find the bound

$$
|\operatorname{Tr} \tau(\omega)|=\left|\operatorname{Tr} \tau(\omega) \operatorname{Tr} \tau_{\infty}^{\prime}(\omega)\right| \leq C_{1} C_{2}^{n} 2^{\nu(\tau)-n_{\mathrm{sp}}(\tau)} .
$$

Applying Eq. (3.9) to the non-identity elements of the sum in Eq. (3.4) gives

$$
\mu_{t}=\frac{1}{e_{t}} \operatorname{dim} \tau^{\prime}+O\left(2^{\nu(\tau)-n_{\mathrm{sp}}(\tau)}\right)
$$

summing this over $t \in T$ gives

$$
\mu(\tau)=\sum_{t \in T} \mu_{t}=\sum_{t \in T} \frac{1}{e_{t}} \operatorname{dim} \tau^{\prime}+O\left(2^{\nu(\tau)-n_{\mathrm{sp}}(\tau)}\right)
$$


Here we apply the "mass formula" (see Vig80, p. 142, Corollaire 2.3):

$$
\sum_{t \in T} \frac{1}{e_{t}}=2^{1-n}\left|\zeta_{K}(-1)\right| h .
$$

Applying this to Eq. (3.11) gives

$$
\mu(\tau)=2^{1-n}\left|\zeta_{K}(-1)\right| h \operatorname{dim} \tau^{\prime}+O\left(2^{\nu(\tau)-n_{\mathrm{sp}}(\tau)}\right) .
$$

As mentioned earlier, it is not necessarily true that $\mu(\tau)=\# S(\tau)$. Indeed, if $v$ is a place at which $\tau_{v}$ is special, then $\mu(\tau)$ counts automorphic representations which are principal series as well as special, owing to the exceptional clause of Theorem 2.1: Types inside of special representations are also contained in principal series representations. We adjust for this possibility using the inclusion-exclusion principle. Let $S_{\mathrm{sp}}$ be the set of places at which $\tau$ is special. For $v \in S_{\mathrm{sp}}$, suppose $\tau_{v}^{\mathrm{ps}} \in \operatorname{Types}\left(K_{v}\right)$ is the type of any principal series representation whose restriction to $\operatorname{GL}\left(2, \mathcal{O}_{K, v}\right)$ contains $\tau_{v}$. Then $\tau_{v}^{\text {ps }}$ is nothing but the central character of $\tau_{v}$.

For each subset $Y$ of $S_{\mathrm{sp}}$, let $\tau^{Y} \in$ Types $(K)$ have the same components as $\tau$, but with $\tau_{v}^{\text {ps }}$ in place of $\tau_{v}$ for each $v \in Y$. Note that for $v \in S_{\mathrm{sp}}$, we have $\operatorname{dim} \tau_{v}=q_{v}$, so that

$$
\operatorname{dim}\left(\tau^{Y}\right)^{\prime}=\operatorname{dim} \tau^{\prime} \prod_{v \in Y} q_{v}^{-1} .
$$

Letting $\kappa=2^{1-n} \zeta_{K}(-1) h$, we have the following expression for $S(\tau)$ :

$$
\begin{aligned}
& S(\tau)=\sum_{Y \subseteq S_{\mathrm{sp}}}(-1)^{\# Y} \mu\left(\tau^{Y}\right) \\
& =\sum_{Y \subseteq S_{\mathrm{sp}}}(-1)^{\# Y}\left(\kappa \operatorname{dim}\left(\tau^{Y}\right)^{\prime}+O\left(2^{\nu(\tau)-\# S_{\mathrm{sp}}}\right)\right) \quad \text { by Eq. (3.12) applied to } \tau^{Y} \\
& =\sum_{Y \subseteq S_{\mathrm{sp}}}(-1)^{\# Y} \kappa \operatorname{dim} \tau^{\prime} \prod_{v \in Y} q_{v}^{-1}+O\left(2^{\nu(\tau)}\right. \\
& =\kappa \operatorname{dim} \tau^{\prime} \prod_{v \in S_{\mathrm{sp}}}\left(1-\frac{1}{q_{v}}\right)+O\left(2^{\nu(\tau)}\right) \\
& =\kappa d(\tau)+O\left(2^{\nu(\tau)}\right)
\end{aligned}
$$

by Eq. (3.1),

thus completing the proof of Theorem 1.1 when $[K: \mathrm{Q}]$ is even.

\section{Proof in the CASE of $[K: \mathbf{Q}]$ OdD}

4.1. Shimura Curves. In this section $n=[K: \mathrm{Q}]$ is odd. Label the infinite places of $K$ as $v_{0}, \ldots, v_{n-1}$. Let $D / K$ be a quaternion 
algebra ramified exactly at $S_{\infty} \backslash\left\{v_{0}\right\}$, let $G$ be the inner form of $\operatorname{GL}(2)_{K}$ corresponding to $D$, and let $\mathcal{O}$ be a maximal order of $D$. Keep the notations $\mathbf{A}$ and $\mathbf{A}_{f}$ from the preceding section. For simplicity we will assume at first that $K \neq \mathbf{Q}$, and then explain how to modify the proof in the case of $K=\mathrm{Q}$ at the end of the section.

Let $X=\mathbf{C} \backslash \mathbf{R}$. For an ideal $N$ of $\mathcal{O}_{K}$, the Shimura curve $X_{D}(N)$ of (full) level $N$ is the one whose complex points are

$$
X_{D}(N)(\mathbf{C})=G(K) \backslash\left(X \times G\left(\mathbf{A}_{f}\right)\right) / U_{N},
$$

where $U_{N} \subset \hat{\mathcal{O}}^{*}$ is the compact open subgroup consisting of elements congruent to the identity modulo $N$. Then $X_{D}(N)$ admits an action of $\hat{\mathcal{O}}^{*} / U_{N} \cong \mathrm{GL}\left(2, \mathcal{O}_{K} / N\right)$.

Now suppose $\tau \in \operatorname{Types}(K)$ is a global type. For $0 \leq i \leq n-1$, suppose that $\tau_{v_{i}} \cong \mathcal{D}_{k_{v}, w}$, and let $\mathbf{k}=\left(k_{0}, \ldots, k_{n-1}\right)$. As in the case of $n$ even, we define a representation $\tau_{f}^{\prime}$ of $\hat{\mathcal{O}}^{*}$ as well as a representation $\tau_{\infty}^{\prime}$ of $G(K \otimes \mathbf{R})$, the only difference being that $\tau_{v_{0}}^{\prime}$ is essentially the same as $\tau_{v_{0}}$ because $G$ is split at that place. Let also $N$ be the level of $\tau_{f}^{\prime}$. As in the previous section, let $\mu(\tau)$ be the number of cuspidal automorphic representations $\pi$ of $G$ containing $\tau=\tau_{f} \otimes \tau_{\infty}$. The strategy is to determine $\mu(\tau)$ by computing the multiplicity of $\tau$ in the appropriate cohomology group of the Shimura curve $X_{D}(N)$.

We proceed as in Car83, 2.1.2, by defining a complex vector bundle $\mathcal{L} / X_{D}(N)$ analytically by

$$
\mathcal{L}=G(K) \backslash\left(X \times G\left(\mathbf{A}_{f}\right) \times V\right) / U_{N} .
$$

Here $V=\bigotimes_{i=0}^{n-1} V_{i}$ is a certain representation of $G(K \otimes \mathbf{R})=\prod_{i=0}^{n-1} G\left(K_{v_{i}}\right)$; for $i>0, V_{i}$ is $\tau_{v}^{\prime}$, while for $i=0, V_{i}$ is a twist of the $(k-2)$ nd symmetric power of the tautological representation of $G\left(K_{v_{0}}\right)=\mathrm{GL}(2, \mathbf{R})$ on $\mathbf{C}^{2}$. Note that the vector bundle $\mathcal{L}$ is equivariant under the action of $\mathrm{GL}(2, \mathcal{O} / N)$, so that the cohomology $H^{1}\left(X_{D}(N), \mathcal{L}\right)$ admits an action of this group. We now appeal to BW00, VII, 3.2 (or for our particular application, see Car83, 2.2.4) to compute the de Rham cohomology of $\mathcal{L}$ :

$$
H^{1}\left(X_{D}(N), \mathcal{L}\right) \cong \bigoplus_{\pi} H^{1}\left(\mathfrak{g}, K_{\infty}, \pi_{\infty} \otimes V\right) \otimes\left(\pi_{f}\right)^{U_{N}}
$$

Here $\mathfrak{g}$ is the Lie algebra of $G(K \otimes \mathbf{R})$ and $K_{\infty} \subset G(K \otimes \mathbf{R})$ is the stabilizer of a point of $X$. The sum runs over cuspidal automorphic representations $\pi$ of $G$ for which the summand is nonzero. It follows from [RT83], Prop. 1.5, that $H^{1}\left(\mathfrak{g}, K_{\infty}, \pi_{\infty} \otimes V\right)$ is zero unless $\pi_{\infty} \cong$ $\tau_{\infty}$, in which case it has dimension 2. The isomorphism in Eq. 4.1 
being $\mathrm{GL}\left(2, \mathcal{O}_{K} / N\right)$-equivariant, we find that

$$
\mu(\tau)=\frac{1}{2}\left\langle\tau_{f}, H^{1}\left(X_{D}(N), \mathcal{L}\right)\right\rangle_{\mathrm{GL}\left(2, \mathcal{O}_{K} / N\right)} .
$$

We now decompose the curve $X_{D}(N)$ into pieces which are stable under the action of $(\mathcal{O} / N \mathcal{O})^{*}$. Let $T=G(K) \backslash G\left(\mathbf{A}_{f}\right) / \hat{\mathcal{O}}^{*}$. There is a bijection between $T$ and the class group $K^{*} \backslash \mathbf{A}_{f}^{*} / \hat{\mathcal{O}}_{K}^{*}$ of $K$, see Vig80, Corollaire 5.7, part (i). We find that

$$
\begin{aligned}
X_{D}(N)(\mathbf{C}) & =\coprod_{t \in T} G(K) \backslash\left(X \times G(K) t \hat{\mathcal{O}}^{*}\right) / U_{N} \\
& =\coprod_{t \in T} \mathcal{O}_{t}^{*} \backslash\left(X \times \hat{\mathcal{O}}^{*}\right) / U_{N},
\end{aligned}
$$

where $\mathcal{O}_{t}=t \hat{\mathcal{O}} t^{-1} \cap D$ is to be considered a subgroup of $\hat{\mathcal{O}}^{*}$ via conjugation by $t$. Write $X_{D, t}(N)$ for $\mathcal{O}_{t}^{*} \backslash\left(X \times \hat{\mathcal{O}}^{*}\right) / U_{N}$; it is a Riemann surface with an action of $\hat{\mathcal{O}}^{*} / U_{N} \cong \mathrm{GL}\left(2, \mathcal{O}_{K} / N\right)$. Suppose $\Gamma_{t}(1)$ is the image in $\operatorname{GL}(2, \mathbf{R})$ of the group of units of $\mathcal{O}_{t}^{*}$ and that $\Gamma_{t}(N) \subset \Gamma_{t}(1)$ arises from the subgroup of elements congruent to 1 modulo $N$. It is easy to check that

$$
X_{D, t}(N)=\hat{\mathcal{O}}^{*} / U_{N} \times_{\Gamma_{t}(1) / \Gamma_{t}(N)} \Gamma_{t}(N) \backslash X .
$$

¿From Eq. 4.3 we have the isomorphism of $\hat{\mathcal{O}}^{*} / U_{N}$-modules

$$
H^{1}\left(X_{D, t}, \mathcal{L}\right)=\operatorname{Ind}_{\Gamma_{t}(1) / \Gamma_{t}(N)} H^{1}\left(\Gamma_{t}(N) \backslash X, \mathcal{L}\right) ;
$$

by abuse of notation we have written $\mathcal{L}$ for the restriction of that vector bundle to both $X_{D, t}(N)$ and $\Gamma_{t}(N) \backslash X$. Let $\mu_{t}$ be the multiplicity of $\tau_{f}^{\prime}$ inside $H^{1}\left(X_{D, t}, \mathcal{L}\right)$ as an $\hat{\mathcal{O}}^{*} / U_{N^{-}}$module, so that by Eq. $4.22 \mu(\tau)=$ $\sum_{t \in T} \mu_{t}$. By Frobenius reciprocity

$$
\mu_{t}=\left\langle\left.\tau_{f}^{\prime}\right|_{\Gamma_{t}(1) / \Gamma_{t}(N)}, H^{1}\left(\Gamma_{t}(N) \backslash X, \mathcal{L}\right)\right\rangle_{\Gamma_{t}(1) / \Gamma_{t}(N)} .
$$

4.2. Cohomology of discrete groups. It therefore remains to compute the structure of $H^{1}\left(\Gamma_{t}(N) \backslash X, \mathcal{L}\right)$ as a $\Gamma_{t}(1) / \Gamma_{t}(N)$-module. Assume that $N$ is large enough so that every nonscalar element of $\Gamma_{t}(N)$ acts without fixed points on $X$. As $\Gamma_{t}(N) \backslash X$ is compact, $H^{1}\left(\Gamma_{t}(N) \backslash X, \mathcal{L}\right) \cong$ $H^{1}\left(\Gamma_{t}(N), V\right)$. It will suffice to compute the Euler characteristic

$$
\chi\left(\Gamma_{t}(N), V\right)=\sum_{i=0}^{2}(-1)^{i}\left[H^{i}\left(\Gamma_{t}(N), V\right)\right]
$$

in the Grothendieck group of $\Gamma_{t}(1) / \Gamma_{t}(N)$. For this we have the: 
Lemma 4.1. Let $\Gamma \subset \mathrm{GL}(2, \mathbf{R})$ be a discrete subgroup, acting on $X$ with compact quotient. Let $x_{1}, \ldots, x_{r} \in X$ be a complete set of $\Gamma$ inequivalent fixed points having fixed subgroups $\Gamma_{1}, \ldots, \Gamma_{r}$. Suppose $\Gamma^{\prime} \subset \Gamma$ is a normal subgroup acting without fixed points on $X$. Let $G=\Gamma / \Gamma^{\prime}$ and let $Z \subset \Gamma$ be the group of elements of $\Gamma$ which are scalar. Suppose $V$ is a finite-dimensional complex vector space admitting an action of $\Gamma$ such that scalar matrices in $\Gamma^{\prime}$ act trivially on $V$. Then we have the equality:

$$
\chi\left(\Gamma^{\prime}, V\right)=\left.(\chi(\Gamma \backslash X)-r) \operatorname{Ind}_{Z}^{G} V\right|_{Z}+\left.\sum_{i=1}^{r} \operatorname{Ind}_{\Gamma_{i}}^{G} V\right|_{\Gamma_{i}}
$$

in the Grothendieck group of $G$.

Proof. We construct a simplicial complex $\mathcal{K}$ with underlying topological space $X$ in such a way that $\Gamma$ acts on $\mathcal{K}$ and such that each elliptic fixed point $x_{j}$ is a vertex of $\mathcal{K}$. For $i=0,1,2$ let $C_{i}(\mathcal{K})$ be the free $C$-vector space with basis the $i$-cells of $K$, and let $N_{i}=\operatorname{dim} C_{i}(\mathcal{K})$. Let $C^{i}(\mathcal{K}, V)$ be the space of $\mathbf{C}\left[\Gamma^{\prime}\right]$-module homomorphisms $C_{i}(\mathcal{K}) \rightarrow V$. Then $C^{i}(\mathcal{K}, V)$ carries a left $\Gamma$-module structure: if $\gamma \in \Gamma$ and $f \in$ $C^{i}(\mathcal{K}, V)$, then $(\gamma f)(x)=\gamma\left(f\left(\gamma^{-1}(y)\right)\right)$. The action factors through an action of $G$. It is standard (see [Shi71], Prop. 8.1) that the cohomology of the complex

$$
0 \rightarrow C^{0}(\mathcal{K}, V) \rightarrow C^{1}(\mathcal{K}, V) \rightarrow C^{2}(\mathcal{K}, V) \rightarrow 0
$$

agrees with $H^{i}\left(\Gamma^{\prime}, V\right)$. Therefore $\chi\left(\Gamma^{\prime}, V\right)$ is the alternating sum of the $C^{i}(\mathcal{K}, V)$ in the Grothendieck ring of $G$.

We wish to compute the structure of $C^{0}(\mathcal{K}, V)$ as an $\mathbf{C}[G]$-module. Let $x_{1}, \ldots, x_{N_{0}}$ be a complete set of $\Gamma$-inequivalent 0 -cells of $\mathcal{K}$, with the fixed points $x_{1}, \ldots, x_{r}$ as in the hypothesis. Writing $W_{i}$ for the $\mathbf{C}$-span of the $\Gamma$-orbit of $x_{i}$, we have a decomposition of $\mathbf{C}[\Gamma]$-modules $C_{0}(\mathcal{K})=\bigoplus_{i=1}^{N_{0}} W_{i}$ of $C_{0}(\mathcal{K})$, and therefore

$$
C^{0}(\mathcal{K}, V)=\bigoplus_{i=1}^{N_{0}} \operatorname{Hom}_{\Gamma^{\prime}}\left(W_{i}, V\right) .
$$

Note that $W_{i} \approx \operatorname{Ind}_{\Gamma_{i}}^{\Gamma} 1$ for $i \leq r$, and that $\Gamma_{i}=Z$ for $i \geq r+1$. For each $i$, the $\mathbf{C}[G]$-module $\operatorname{Hom}_{\Gamma^{\prime}}\left(W_{i}, V\right)$ can be modeled on the space of functions $\Phi: \Gamma \rightarrow V$ satisfying $\Phi\left(\gamma^{\prime} \gamma k\right)=\rho\left(\gamma^{\prime}\right) \Phi(\gamma)$ whenever $\gamma^{\prime} \in \Gamma^{\prime}$, $\gamma \in \Gamma, k \in \Gamma_{i}$. The action of $G$ is given by $\bar{g}(\Phi)(\gamma)=\rho(g) \Phi\left(g^{-1} \gamma\right)$ whenever $\bar{g}=\Gamma^{\prime} g \in G$.

We claim that there is an isomorphism of $G$-modules

$$
\left.\operatorname{Hom}_{\Gamma^{\prime}}\left(W_{i}, V\right) \stackrel{\sim}{\longrightarrow} \operatorname{Ind}_{\Gamma_{i}}^{G} V\right|_{\Gamma_{i}} .
$$


The space on the right is modeled on the space of functions $\Psi: G \rightarrow V$ satisfying $\Psi\left(k g \Gamma^{\prime}\right)=\rho(k) \Psi\left(g \Gamma^{\prime}\right)$ for all $k \in \Gamma_{i}, g \Gamma \in G$. Choose a set of representatives $g_{1}, \ldots, g_{s}$ for $G / \Gamma_{i}=\Gamma^{\prime} \backslash \Gamma / \Gamma_{i}$; it is easily checked that an isomorphism is given by $\Phi \mapsto \Psi$, where $\Psi\left(\Gamma^{\prime} g_{j}\right)=g_{j} \Phi\left(g_{j}^{-1}\right)$.

Thus in the Grothendieck group of $G$ we have

$$
\begin{aligned}
C^{0}(\mathcal{K}, V) & =\left.\sum_{i=1}^{N_{0}} \operatorname{Ind}_{\Gamma_{i}}^{G} V\right|_{\Gamma_{i}} \\
& =\left.\left(N_{0}-r\right) \operatorname{Ind}_{Z}^{G} V\right|_{Z}+\left.\sum_{i=1}^{r} \operatorname{Ind}_{\Gamma_{i}}^{G} V\right|_{\Gamma_{i}} .
\end{aligned}
$$

A similar calculation holds for $C^{1}(\mathcal{K}, V)$ and $C^{2}(\mathcal{K}, V)$, except that there are no cells fixed by elements of $\Gamma$. Therefore:

$$
\begin{aligned}
& C^{1}(\mathcal{K}, V)=N_{1} \operatorname{Ind}_{Z}^{G} V \\
& C^{2}(\mathcal{K}, V)=N_{2} \operatorname{Ind}_{Z}^{G} V
\end{aligned}
$$

Taking the alternating sum of the $C^{i}(\mathcal{K}, V)$ gives the expression in the Lemma.

We apply Lemma 4.1 to the groups $\Gamma_{t}(N) \subset \Gamma_{t}(1)$ and to the representation $V$ of $\Gamma_{t}(1)$. Keep the notations $\Gamma_{i}, x_{i}$, and $r$ from the lemma. Since the multiplicity of $\tau$ in $H^{0}$ and $H^{2}$ is bounded, the multiplicity of $\tau$ in $\bigoplus_{t \in T} \chi\left(\Gamma_{t}(N), V\right)$ is $-\sum_{t \in T} \mu_{t}=-2 \mu(\tau)$ to within an error term depending only on $K$. From Eq. (4.4) we have

$$
-\mu_{t}=\left(\chi\left(X_{t}(1)\right)-r\right) \operatorname{dim} V \operatorname{dim} \tau_{f}^{\prime}+\sum_{i=1}^{r}\left\langle\left.\tau_{f}^{\prime}\right|_{\Gamma_{i}},\left.V\right|_{\Gamma_{i}}\right\rangle+O(1)
$$

Let $W_{i}=\Gamma_{i} / Z$ have order $e_{i}$. Since $\tau_{f}^{\prime}$ and $V$ have the same values on $Z=\mathcal{O}_{K}^{*}$, the summand on the right is

$$
\left\langle\left.\tau_{f}^{\prime}\right|_{\Gamma_{i}},\left.V\right|_{\Gamma_{i}}\right\rangle=\frac{1}{e_{i}} \sum_{w \in W_{i}} \operatorname{Tr} \tau_{f}^{\prime}(w) \overline{\operatorname{Tr}(w \mid V)} .
$$

By an argument of the same sort as in the previous section, this quantity is $\frac{1}{e_{i}} \operatorname{dim} \tau_{f}^{\prime} \operatorname{dim} V+O\left(2^{\nu(\tau)-n_{\mathrm{sp}}(\tau)}\right)$. Therefore

$$
-2 \mu_{t}=\left[\chi\left(X_{h}(1)\right)-r+\sum_{i=1}^{r} \frac{1}{e_{i}}\right] \operatorname{dim} \tau_{f}^{\prime} \operatorname{dim} V+O\left(2^{\nu(\tau)-n_{\mathrm{sp}}(\tau)}\right) .
$$


The expression in square brackets is

$$
\begin{aligned}
\chi\left(X_{t}(1)\right)-\sum_{i=1}^{r}\left(1-\frac{1}{e_{i}}\right) & =-\frac{1}{2 \pi} \operatorname{vol}\left(\Gamma_{t}(1) \backslash X\right) \\
& =-\left|\zeta_{K}(-1)\right| 2^{2-n}
\end{aligned}
$$

from [Vig80, p. 109, Exemple 5, together with p. 119, Prop. 2.10. Since $2 \mu(\tau)=\sum_{t \in T} \mu_{t}$ and $\# T=h$, the argument continues exactly as in the previous section.

4.3. The case of $K=\mathrm{Q}$. Now suppose $K=\mathbf{Q}$. Let $\tau=\tau_{f} \otimes \tau_{\infty} \in$ Types $(\mathbf{Q})$. Let $N$ be the level of $\tau$, considered as a rational integer, and suppose the infinite component of $\tau$ is $\tau_{\infty}=D_{k, w}$. Assume $N \geq 22$. We note that condition (2) in the definition of global types reduces to the condition that the central character of $\tau_{f}$ take the value $(-1)^{k}$ at -1 .

Let $\mu(\tau)$ be the number of cuspidal automorphic representations $\pi$ of $\operatorname{GL}\left(2, \mathbf{A}_{\mathbf{Q}}\right)$ containing $\tau=\tau_{f} \otimes \tau_{\infty}$. The analogue of Eq. 4.1 in the case of $K=\mathrm{Q}$ is found in [Lan73], Thm. 2.10. The result is the same except that parabolic cohomology must be used. Let $Y_{D}(N)$ be the (non-complete) Shimura curve for the split algebra $D=M_{2}(\mathbf{Q})$. Let $\mathcal{L} / Y_{D}(N)$ be the vector bundle corresponding to $\tau_{\infty}$; then

$$
\mu(\tau)=\frac{1}{2}\left\langle\tau_{f}, H_{P}^{1}\left(X_{D}(N), \mathcal{L}\right)\right\rangle_{\mathrm{GL}(2, \mathbf{Z} / N \mathbf{Z})} .
$$

The curve $Y_{D}(N)$ has connected components, each of which is the classical modular curve $Y(N)$ :

$$
Y_{D}(N)=\mathrm{GL}(2, \mathbf{Z} / N \mathbf{Z}) \times_{\mathrm{SL}(2, \mathbf{Z} / N \mathbf{Z})} Y(N) .
$$

Therefore

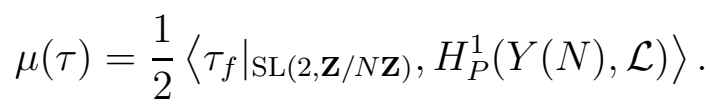

Our goal is therefore to determine the class of $H_{P}^{1}(Y(N), \mathcal{L})$ in the Grothendieck group of $\mathrm{SL}(2, \mathbf{Z} / N \mathbf{Z})$. Since $N>1$, the group $\Gamma(N)$ acts on the upper half-pane $\mathcal{H}$ without fixed points and we have the $S_{n}$-equivariant isomorphism

$$
H_{P}^{1}(Y(N), \mathcal{L}) \cong H_{P}^{1}\left(\Gamma(N), V_{k}\right),
$$

where $V_{k}=\operatorname{Sym}^{k-2} V_{3}$ is the $(k-2)$ nd symmetric power of the tautological representation of $\mathrm{SL}(2, \mathbf{Z})$ on $\mathbf{C}^{2}$. (For the definition of parabolic cohomology of a Fuchsian group, see [Shi71], 8.1.)

We now compute the $S_{N}$-module $H_{P}^{1}\left(\Gamma(N), V_{k}\right)$. The calculation hinges on the geometry of the Galois cover $X(N) \rightarrow X(1)$ of (complete) modular curves. Let $S_{N}=\operatorname{SL}(2, \mathbf{Z} / N \mathbf{Z})$; then the Galois group of this 
cover is $S_{N} /\{ \pm I\}$. The cover is branched over three points in $X(1)$, namely the images in $X(1)$ of the points $\rho=e^{2 \pi i / 3}, i$, and $\infty$ of the upper half plane $\mathcal{H}$. Those points have the following stabilizers in $\operatorname{SL}(2, \mathbf{Z})$ :

$$
\begin{aligned}
\Gamma_{\rho} & =\left\langle\left(\begin{array}{cc}
0 & -1 \\
1 & 1
\end{array}\right)\right\rangle \\
\Gamma_{i} & =\left\langle\left(\begin{array}{cc}
0 & -1 \\
1 & 0
\end{array}\right)\right\rangle \\
\Gamma_{\infty} & =\left\langle \pm\left(\begin{array}{ll}
1 & 1 \\
& 1
\end{array}\right)\right\rangle .
\end{aligned}
$$

For $j \in\{\rho, i, \infty\}$, let $\bar{\Gamma}_{j}$ be the image of $\Gamma_{j}$ in $S_{N}$. The center of $\mathrm{SL}(2, \mathbf{Z})$ is $Z=\{ \pm 1\}$; let $\bar{Z}$ be its image in $S_{N}$. Since $N>2$, the reduction map $Z \rightarrow \bar{Z}$ is an isomomorphism, and therefore any $Z$ module can be considered an $\bar{Z}$-module. The same is true for the groups $\Gamma_{j}$ and $\bar{\Gamma}_{j}$.

To compute the structure of $H_{P}^{1}\left(\Gamma(N), V_{k}\right)$ as an $S_{N}$-module, we must modify Lemma 4.1 to include a term coming from the unique cusp of $X(1)$. Let $\chi_{P}\left(\Gamma(N), V_{k}\right)$ be the alternating sum of the parabolic cohomology groups $H_{P}^{i}\left(\Gamma(N), V_{k}\right)$ in the Grothendieck group of $S_{N}$. Let sgn denote the obvious character of $\bar{Z}$ and of $\bar{\Gamma}_{\infty}$.

Lemma 4.2.

$\chi_{P}\left(\Gamma(N), V_{k}\right)=-\left[\left.\operatorname{Ind}_{\bar{Z}}^{S_{N}} V_{k}\right|_{Z}\right]+\left[\left.\operatorname{Ind}_{\bar{\Gamma}_{\rho}}^{S_{N}} V_{k}\right|_{\Gamma_{\rho}}\right]+\left[\left.\operatorname{Ind}_{\bar{\Gamma}_{i}}^{S_{N}} V_{k}\right|_{\Gamma_{i}}\right]+\left[\operatorname{Ind}_{\bar{\Gamma}_{\infty}}^{S_{N}} \operatorname{sgn}^{k}\right]$

Proof. Remove a small open disc containing $\infty$ from the projective line $X(1)$, and let $\mathcal{H}^{o}$ be the preimage of the result. This can be accomplished by removing from $\mathcal{H}$ all the $\mathrm{SL}(2, \mathbf{Z})$-translates of the region $\left\{x+i y \mid y \geq y_{0}\right\}$ for $y_{0}$ large enough. Construct an $\mathrm{SL}(2, \mathbf{Z})$-stable simplicial complex $\mathcal{K}$ whose underlying topological space is $\mathcal{H}^{o}$ such that $i$ and $\rho$ are vertices of $\mathcal{K}$. Let $u=\left(\begin{array}{ll}1 & 1 \\ 0 & 1\end{array}\right)$, so that $\Gamma_{\infty} \cap \Gamma(N)$ is generated by $u^{N}$. Assume there is a 1 -cell $t_{1}$ in $\mathcal{K}$ for which $\partial t_{1}$ is of the form $(u-1) z$ for a vertex $z$ of $\mathcal{K}$. Then the 1 -chain $\gamma:=\sum_{j=0}^{N-1} u^{j} t_{1}$ has boundary $\left(u^{N}-1\right) z$. The boundary of the quotient complex $\Gamma(N) \backslash \mathcal{K}$ is exactly the set of translates $g \gamma$, where $g$ runs over a set of coset representatives for $\Gamma(N) \backslash \operatorname{SL}(2, \mathbf{Z})$. Note that $\partial(g \gamma)=\left(g u^{N} g^{-1}-1\right) g z$. Let $C_{P}^{1}\left(\mathcal{K}, V_{k}\right) \subset C^{1}\left(\mathcal{K}, V_{k}\right)$ be the space of $\mathbf{C}[\Gamma(N)]$-module homomorphisms $C_{1}(\mathcal{K}) \rightarrow V_{k}$ mapping the 1-cycle $g \gamma$ into $\left(g u^{N} g^{-1}-1\right) V_{k}$ for each $g \in \mathrm{SL}(2, \mathbf{Z})$. Then the complex

$$
1 \rightarrow C^{0}\left(\mathcal{K}, V_{k}\right) \rightarrow C_{P}^{1}\left(\mathcal{K}, V_{k}\right) \rightarrow C^{2}\left(\mathcal{K}, V_{k}\right) \rightarrow 1
$$


has cohomology $H^{*}\left(\Gamma(N), V_{k}\right)$ (loc. cit., Prop. 8.1). We remark that $\left(u^{N}-1\right) V_{k}=(u-1) V_{k}$ has codimension 1 in $V_{k}$.

Let $t_{1}, t_{2}, \ldots, t_{N_{1}}$ be a complete set of $\operatorname{SL}(2, \mathbf{Z})$-inequivalent 1-cells of $\mathcal{K}$. Writing $W_{j}$ for the $\mathbf{C}$-span of the $\operatorname{SL}(2, \mathbf{Z})$-orbit of $t_{j}$, we have an isomorphism of $\mathrm{SL}(2, \mathbf{Z})$-modules $W_{j} \cong \operatorname{Ind}_{Z}^{\mathrm{SL}(2, \mathbf{Z})} 1$. Let $C_{P}^{1}\left(W_{j}, V_{k}\right) \subset$ $C_{P}^{1}\left(\mathcal{K}, V_{k}\right)$ be the $\mathrm{SL}(2, \mathbf{Z})$-submodule of cocycles supported on $W_{j}$, so that $C_{1}^{P}\left(\mathcal{K}, V_{k}\right)=\bigoplus_{j=1}^{N_{1}} C_{P}^{1}\left(W_{j}, V_{k}\right)$. Then for $j>1$ we have

$$
\left[C^{P}\left(W_{j}, V_{k}\right)\right]=\left[\operatorname{Hom}_{\Gamma(N)}\left(W_{j}, V_{k}\right)\right]=\left[\left.\operatorname{Ind}_{\bar{Z}}^{S_{N}} V_{k}\right|_{Z}\right]
$$

as classes in the Grothendieck group of $S_{N}$. On the other hand for $j=1$ we have the exact sequence of $S_{N}$-modules

$0 \rightarrow C_{P}^{1}\left(W_{1}, V_{k}\right) \rightarrow \operatorname{Hom}_{\Gamma(N)}\left(W_{j}, V_{k}\right) \stackrel{\xi}{\rightarrow} \operatorname{Hom}_{\bar{\Gamma}_{\infty}}\left(\mathbf{C}\left[S_{N}\right], V_{k} /(u-1) V_{k}\right) \rightarrow 0$

where $\xi(f): \mathbf{C}\left[S_{N}\right] \rightarrow V_{k} /(u-1) V_{k}$ is defined by $\xi(f)(\bar{g})=g^{-1} f(g \gamma)+$ $(u-1) V_{k}$. The homomorphism $\xi(f)$ is $\bar{\Gamma}_{\infty}$-equivariant because

$$
\begin{aligned}
\xi(f)(g u) & =u^{-1} g^{-1} f(g u \gamma) \\
& =u^{-1} g^{-1} f(g u \gamma) \\
& =u^{-1} g^{-1} f\left(g\left(u^{N}-1\right) t_{1}+g \gamma\right) \\
& =u^{-1} g^{-1}\left(u^{N}-1\right) f\left(g t_{1}\right)+u^{-1} g^{-1} f(g \gamma) \text { because } u^{N}-1 \in \mathbf{C}[\Gamma(N)] \\
& \equiv g^{-1} f(g \gamma) \equiv \xi(f)(g) \quad\left(\bmod (u-1) V_{k}\right) .
\end{aligned}
$$

Since $\left[V_{k} /(u-1) V_{k}\right]=\left[\operatorname{sgn}^{k}\right]$ in the Grothendieck group of $\bar{\Gamma}_{\infty}$, Eq. 4.9 implies that

$$
\left[C_{P}^{1}\left(W_{1}, V_{k}\right)\right]=\left[\left.\operatorname{Ind}_{\bar{Z}}^{S_{N}} V_{k}\right|_{Z}\right]-\left[\operatorname{Ind}_{\bar{\Gamma}_{\infty}}^{S_{N}} \operatorname{sgn}^{k}\right]
$$

and therefore that

$$
\left[C_{P}^{1}\left(\mathcal{K}, V_{k}\right)\right]=\bigoplus_{j=1}^{N_{1}}\left[C_{P}^{1}\left(W_{j}, V_{k}\right)\right]=N_{1}\left[\left.\operatorname{Ind}_{\bar{Z}}^{S_{N}} V_{k}\right|_{Z}\right]-\left[\operatorname{Ind}_{\bar{\Gamma}_{\infty}}^{S_{N}} \operatorname{sgn}^{k}\right]
$$

in the Grothendieck group of $S_{N}$.

Therefore the calculation of $\chi\left(\Gamma(N), V_{k}\right)$ proceeds as in Lemma 4.1 with the only change being that there is a contribution of $\left[\operatorname{Ind}_{\bar{\Gamma}_{\infty}}^{S_{N}} \operatorname{sgn}^{k}\right]$ coming from the space of 1-cochains. Since $\chi\left(\operatorname{SL}(2, \mathbf{Z}) \backslash \mathcal{H}^{o}\right)=1$ and there are two $\mathrm{SL}(2, \mathbf{Z})$-orbits of elliptic fixed points, the appropriate modification of Eq. 4.5 is

$$
\chi\left(\Gamma(N), V_{k}\right)=-\left[\left.\operatorname{Ind} \frac{S_{N}}{Z} V_{k}\right|_{Z}\right]+\sum_{j \in\{\rho, i\}}\left[\left.\operatorname{Ind} \frac{S_{N}}{\Gamma_{j}} V_{k}\right|_{\Gamma_{j}}\right]+\left[\operatorname{Ind}_{\bar{\Gamma}_{\infty}}^{S_{N}} \operatorname{sgn}^{k}\right]
$$

as required. 
The relationship between $H_{P}^{1}\left(\Gamma(N), V_{k}\right)$ and $\chi_{P}\left(\Gamma(N), V_{k}\right)$ is given by

$$
\left[H_{P}^{1}\left(\Gamma(N), V_{k}\right)\right]= \begin{cases}-\chi_{P}\left(\Gamma(N), V_{k}\right)-2[1], & k=2 \\ -\chi_{P}\left(\Gamma(N), V_{k}\right), & k>2\end{cases}
$$

the reason being that both $H_{P}^{0}\left(\Gamma(N), V_{k}\right)$ and $H_{P}^{2}\left(\Gamma(N), V_{k}\right)$ are onedimensional if $k=2$ and vanish if $k>2$. We analyze each of the three terms appearing on the right-hand side of Eq. 4.2. For the first term, note that $\left.V_{k}\right|_{Z}$ is simply $k-1$ copies of the sign character $\operatorname{sgn}^{k}$ of $Z$, so that

$$
\left[\left.\operatorname{Ind}_{\bar{Z}}^{S_{N}} V_{k}\right|_{Z}\right]=(k-1)\left[\operatorname{Ind}_{\bar{Z}}^{S_{N}} \operatorname{sgn}^{k}\right] .
$$

For the second term of Eq. 4.2, we have $\left.V_{3}\right|_{\Gamma_{\rho}}=\chi_{\rho} \oplus \chi_{\rho}^{-1}$, where $\chi_{\rho}: \Gamma_{\rho} \rightarrow \mathbf{C}^{*}$ is the character $\left(\begin{array}{cc}-1 \\ 1 & 1\end{array}\right) \mapsto e^{2 \pi i / 6}$. Then

$$
\left.V_{k}\right|_{\Gamma_{\rho}}=\left.\operatorname{Sym}^{k-2} V_{3}\right|_{\Gamma_{\rho}}=\chi_{\rho}^{k-2} \oplus \chi_{\rho}^{k-4} \oplus \cdots \oplus \chi_{\rho}^{-k+2}
$$

Each character of $\Gamma_{\rho}$ of the same parity as $k$ appears in the sum about $k / 3$ times, so we expect the right side of Eq. 4.12 to contain about $k / 3$ copies of the sum $\chi_{\rho}^{k} \oplus \chi_{\rho}^{k+2} \oplus \chi_{\rho}^{k+4}=\operatorname{Ind}_{Z}^{\Gamma_{\rho}} \operatorname{sgn}^{k}$. More precisely, we have the following relation in the Grothendieck group of $\Gamma_{\rho}$ :

$$
\left.V_{k}\right|_{\Gamma_{\rho}}=\left\lfloor\frac{k}{3}\right\rfloor\left[\operatorname{Ind}_{Z}^{\Gamma_{\rho}} \operatorname{sgn}^{k}\right]+\varepsilon_{\rho}
$$

where the error term is a virtual representation of $\Gamma_{\rho}$ given by

$$
\varepsilon_{\rho}=\left\{\begin{array}{lll}
-[1], & k \equiv 0 & (\bmod 6) \\
0, & k \equiv 1 & (\bmod 6) \\
{[1],} & k \equiv 2 & (\bmod 6) \\
-\left[\chi_{\rho}^{3}\right], & k \equiv 3 & (\bmod 6) \\
0, & k \equiv 4 & (\bmod 6) \\
{\left[\chi_{\rho}^{3}\right],} & k \equiv 5 & (\bmod 6) .
\end{array}\right.
$$

For the third term of Eq. 4.2, let $\chi_{i}: \Gamma_{i} \rightarrow \mathbf{C}^{*}$ be the character defined by $\left(\begin{array}{cc}-1 & 1\end{array}\right) \rightarrow i$. The analysis is similar to the case of $\Gamma_{\rho}$. We have

$$
\left.V\right|_{\Gamma_{i}}=\left\lfloor\frac{k}{2}\right\rfloor\left[\operatorname{Ind}_{Z}^{\Gamma_{i}} \operatorname{sgn}^{k}\right]+\varepsilon_{i}
$$


where the error term is

$$
\varepsilon_{i}=\left\{\begin{array}{lll}
-[1], & k \equiv 0 & (\bmod 4) \\
0, & k \equiv 1 & (\bmod 4) \\
-\left[\chi_{i}^{2}\right], & k \equiv 2 & (\bmod 4) \\
0, & k \equiv 3 & (\bmod 4)
\end{array}\right.
$$

Let $f(k)=(k-1)-\left\lfloor\frac{k}{3}\right\rfloor-\left\lfloor\frac{k}{2}\right\rfloor$. Substituting Eqs. 4.11, 4.13, and 4.15 into Eq. 4.2 gives:

Theorem 4.3. For $k=2$ we have

$\left[H_{P}^{1}\left(\Gamma(N), V_{2}\right)\right]-2[1]=\left[\operatorname{Ind}_{\bar{Z}}^{S_{N}} 1\right]-\left[\operatorname{Ind}_{\bar{\Gamma}_{\rho}}^{S_{N}} 1\right]-\left[\operatorname{Ind}_{\bar{\Gamma}_{i}}^{S_{N}} 1\right]-\left[\operatorname{Ind}_{\bar{\Gamma}_{\infty}}^{S_{N}} 1\right]$.

For weight $k>2$,

$\left[H_{P}^{1}\left(\Gamma(N), V_{k}\right)\right]=f(k)\left[\operatorname{Ind}_{\bar{Z}}^{S_{N}} \operatorname{sgn}^{k}\right]-\left[\operatorname{Ind}_{\bar{\Gamma}_{\rho}}^{S_{N}} \varepsilon_{\rho}\right]-\left[\operatorname{Ind}_{\bar{\Gamma}_{i}}^{S_{N}} \varepsilon_{i}\right]-\left[\operatorname{Ind}_{\bar{\Gamma}_{\infty}}^{S_{N}} \operatorname{sgn}^{k}\right]$.

We may now complete the proof of Theorem 1.1. Let $\tau \in \operatorname{Types}(\mathbf{Q})$ be a type of level $N$ and weight $k$ and let $\mu(\tau)$ be its multiplicity in $H_{P}^{1}\left(\Gamma(N), V_{k}\right)$. We claim that $\mu(\tau)=\frac{1}{6}(k-1) \operatorname{dim} \tau_{f}+O\left(2^{\nu(\tau)-n_{\mathrm{sp}}(\tau)}\right)$. (Note that $f(k) \sim(k-1) / 6=2 \zeta_{\mathbf{Q}}(-1)(k-1)$.) The only feature separating this calculation from that of the previous section is the appearance of the term $\operatorname{Ind}_{\bar{\Gamma}_{\infty}}^{S_{N}} \operatorname{sgn}^{k}$. By the Chinese remainder theorem the multiplicity of $\tau_{f}$ in this term is a product of local multiplicities $\left\langle\left.\tau_{p}\right|_{U_{p}}, 1\right\rangle$, where $U_{p} \subset \mathrm{GL}\left(2, \mathbf{Z}_{p}\right)$ is the unipotent subgroup. A case-bycase analysis shows that the local multiplicity at $p$ is 1 if $\tau_{p}$ is special and at most 2 in any case. (In fact it is 0 if $\tau_{p}$ is supercuspidal.) Therefore the multiplicity of $\tau_{f}$ in $\operatorname{Ind}_{\bar{\Gamma}_{\infty}}^{S_{N}} \operatorname{sgn}^{k}$ is $O\left(2^{\nu(\tau)-n_{\mathrm{sp}}(\tau)}\right)$. The claim follows and the calculation proceeds exactly as before.

We remark without proof that $\left[H^{1}\left(\Gamma(N), V_{k}\right)\right]=2\left[S_{k}(\Gamma(N))\right]$, where $S_{k}(\Gamma(N))$ is the space of cusp forms of weight $k$ for $\Gamma(N)$.

We conclude the section with a table of types $\tau \in \operatorname{Types}(\mathbf{Q})$ for which $S(\tau)=\emptyset$. Table 1 lists configurations of finite components $\tau_{p}$ together with those weights $k$ of the appropriate parity for which $\tau=\left(\bigotimes_{p} \tau_{p}\right) \otimes \mathcal{D}_{k,(-1)^{k}}$ is a global type without any matching cuspforms. Unfortunately, the list omits local 2-adic types of conductor $2^{n}$, where $n \geq 5$ is odd; the presence of extraordinary (non-dihedral) Galois representations of $\mathbf{Q}_{2}$ in that case complicates matters considerably. The list is complete in the sense that any type $\tau \in \operatorname{Types}(\mathbf{Q})$ with $S(\tau)=\emptyset$ is a twist of one of the listed types, unless that type 
should include one of the aforementioned 2-adic types. The notations for local components are given in Table 2.

\section{The Field OVer Which $J_{1}\left(p^{n}\right)$ IS SEMI-STABLE}

The existence of modular forms with prescribed ramification behavior has arithmetic consequences for the Jacobians of Shimura curves. For simplicity we restrict our attention to the case of $K=\mathrm{Q}$; we examine the modular Jacobians $J=J_{1}\left(p^{n}\right)$ for $p \geq 3$ prime.

In [Kri96 an explicit extension $M$ of $\mathbf{Q}_{p}^{\text {nr }}$ is constructed over which $J_{0}\left(p^{n}\right)$ becomes semi-stable. The result of this section is a converse to this sort of theorem, whereby we construct an explicit extension of $\mathbf{Q}_{p}^{\mathrm{nr}}$ which contains any other field over which $J_{1}\left(p^{n}\right)$ becomes semi-stable.

Following the notations of [Kri96], let $\Omega_{i} / \mathbf{Q}_{p}, i=1,2,3$ be the three quadratic extensions of $\mathbf{Q}_{p}$, with $\Omega_{1} / \mathbf{Q}_{p}$ unramified. One realization of this scenario is $\Omega_{1}=\mathbf{Q}_{p}(\sqrt{D}), \Omega_{2}=\mathbf{Q}_{p}(\sqrt{p}), \Omega_{3}=\mathbf{Q}_{p}(\sqrt{D p})$, where $D \in \mathbf{Z}_{p}^{*}$ is a quadratic nonresidue. For each $i$ let $\mathfrak{p}_{i}$ be the maximal ideal of $\Omega_{i}$ and let $M_{i} / \Omega_{i}^{\mathrm{nr}}$ be the class field with norm subgroup $U_{i}$ defined by

$$
U_{i}= \begin{cases} \pm\left(1+\mathfrak{p}_{i}^{\lfloor n / 2\rfloor}\right) & i=1 \\ 1+\mathfrak{p}_{i}^{n-1}, & i=2,3 .\end{cases}
$$

Finally let $M=M_{1} M_{2} M_{3} \mathbf{Q}_{p}^{\mathrm{nr}}\left(\zeta_{p}^{n}\right)$.

Let $A_{n}$ denote the set of two-dimensional Weil-Deligne representations $\rho_{p}$ of the Weil group of $\mathbf{Q}_{p}$ of conductor dividing $p^{n}$ and satisfying $\operatorname{det} \rho_{p}(-1)=1$. Then $M$ has the following interpretation:

$$
\left.\bigcap_{\rho \in A_{n}} \operatorname{ker} \rho_{p}\right|_{I_{\mathbf{Q}_{p}}} \text { has fixed field precisely } M \text {. }
$$

Indeed, any $\rho_{p} \in A_{n}$ has one of the following forms:

(1) decomposable as $\varepsilon_{1} \oplus \varepsilon_{2}$, where the $\varepsilon_{i}$ have conductor dividing $p^{n}$

(2) $\varepsilon \otimes \operatorname{Sp}(2)$, where $\varepsilon$ has conductor dividing $p^{n}$, or

(3) $\operatorname{Ind}_{\Omega_{i} / \mathbf{Q}_{p}} \theta$, where $i \in\{1,2,3\}$.

In the last case, the condition that $\rho_{p}$ has conductor dividing $p^{n}$ translates into the condition that $\theta$ has conductor $\lfloor n / 2\rfloor$ if $i=1$ and $n-1$ if $i=2,3$, as can be determined from the classification in Section 2.2. The condition that $\operatorname{det} \rho_{p}(-1)=1$ means that $\theta(-1)=1$ if $i=1$, and $\theta(-1)=(-1)^{(p-1) / 2}$ if $i=2,3$. For a given $i$, the fixed field of $\left.\rho_{p}\right|_{I_{\mathbf{Q}_{p}}}$ for $\rho_{p}$ arising from such a character $\theta$ of $\Omega_{i}^{*}$ is exactly $M_{i}$. The claim in Eq. 5.1 follows. 
TABLE 1. Global inertial types over $\mathbf{Q}$ lacking representation by a cusp form. For explanation of notation, see Table 2 .

\begin{tabular}{|c|c|c|c|c|c|}
\hline Cond. & Local Components & $k$ & Cond. & Local Components & $k$ \\
\hline 1 & & $2,4,6,8,10,14$ & 25 & $\mathrm{SC}_{5}(12)$ & 2 \\
\hline 2 & $\mathrm{St}_{2}$ & $2,4,6$ & 26 & $\mathrm{St}_{2}, \mathrm{PS}_{13}(6)$ & 2 \\
\hline \multirow[t]{2}{*}{3} & $\mathrm{St}_{3}$ & 2,4 & 27 & $\mathrm{SC}_{27}(\sqrt{3}, 1)$ & 2 \\
\hline & $\mathrm{PS}_{3}(2)$ & 3,5 & 28 & $\mathrm{SC}_{2}(3), \mathrm{St}_{7}$ & 2 \\
\hline \multirow[t]{2}{*}{4} & $\mathrm{SC}_{2}(3)$ & $2,4,8$ & 36 & $\mathrm{SC}_{2}(3), \mathrm{SC}_{3}(4)$ & 4 \\
\hline & $\mathrm{PS}_{4}$ & 3 & & $\mathrm{SC}_{2}(3), \mathrm{SC}_{3}(8)$ & 3 \\
\hline \multirow[t]{3}{*}{5} & $\mathrm{St}_{5}$ & 2 & 45 & $\mathrm{SC}_{3}(4), \mathrm{St}_{5}$ & 2 \\
\hline & $\mathrm{PS}_{5}(2)$ & 2,4 & 49 & $\mathrm{SC}_{7}(8)$ & 2 \\
\hline & $\mathrm{PS}_{5}(4)$ & 3 & & $\mathrm{SC}_{7}(24)$ & 2 \\
\hline 6 & $\mathrm{St}_{2}, \mathrm{St}_{3}$ & 2 & 50 & $\mathrm{St}_{2}, \mathrm{SC}_{5}(8)$ & 5 \\
\hline \multirow[t]{3}{*}{7} & $\mathrm{St}_{7}$ & 2 & & $\mathrm{St}_{2}, \mathrm{SC}_{5}(24)$ & 3 \\
\hline & $\mathrm{PS}_{7}(3)$ & 2 & 52 & $\mathrm{SC}_{2}(3), \mathrm{PS}_{13}(2)$ & 2 \\
\hline & $\mathrm{PS}_{7}(6)$ & 3 & 54 & $\mathrm{St}_{2}, \mathrm{SC}_{27}(\sqrt{-3}, 1)$ & 3 \\
\hline \multirow[t]{2}{*}{8} & $\mathrm{PS}_{8}$ & 2 & 60 & $\mathrm{SC}_{2}(3), \mathrm{St}_{3}, \mathrm{St}_{5}$ & 2 \\
\hline & $\mathrm{SC}_{8}$ & 2 & 64 & $\mathrm{SC}_{64}(3)$ & 2 \\
\hline \multirow[t]{3}{*}{9} & $\mathrm{SC}_{3}(4)$ & 2,6 & & $\mathrm{SC}_{64}(2)$ & 3 \\
\hline & $\mathrm{SC}_{3}(8)$ & 3 & 72 & $\mathrm{SC}_{8}, \mathrm{SC}_{3}(4)$ & 2 \\
\hline & $\mathrm{PS}_{9}$ & 2 & 90 & $\mathrm{St}_{2}, \mathrm{SC}_{3}(4), \mathrm{PS}_{5}(2)$ & 2 \\
\hline \multirow[t]{2}{*}{10} & $\mathrm{St}_{2}, \mathrm{St}_{5}$ & 2 & & $\mathrm{St}_{2}, \mathrm{SC}_{3}(8), \mathrm{St}_{5}$ & 3 \\
\hline & $\mathrm{St}_{2}, \mathrm{PS}_{5}(2)$ & 2 & 98 & $\mathrm{St}_{2}, \mathrm{SC}_{7}(4)$ & 2 \\
\hline 11 & $\mathrm{PS}_{11}(5)$ & 2 & & $\mathrm{St}_{2}, \mathrm{SC}_{7}(12)$ & 2 \\
\hline 12 & $\mathrm{SC}_{2}(3), \mathrm{St}_{3}$ & 2,6 & 100 & $\mathrm{SC}_{2}(3), \mathrm{SC}_{5}(3)$ & 2 \\
\hline \multirow[t]{3}{*}{13} & $\mathrm{St}_{13}$ & 2 & & $\mathrm{SC}_{2}(3), \mathrm{SC}_{5}(6)$ & 2 \\
\hline & $\mathrm{PS}_{13}(2)$ & 2 & & $\mathrm{SC}_{2}(3), \mathrm{SC}_{5}(8)$ & 3 \\
\hline & $\mathrm{PS}_{13}(3)$ & 2 & & $\mathrm{SC}_{2}(3), \mathrm{SC}_{5}(12)$ & 2 \\
\hline 14 & $\mathrm{St}_{2}, \mathrm{PS}_{7}(3)$ & 2 & 108 & $\mathrm{SC}_{2}(3), \mathrm{SC}_{27}(\sqrt{3}, 1)$ & 2 \\
\hline 15 & $\mathrm{St}_{3}, \mathrm{PS}_{5}(2)$ & 2 & 121 & $\mathrm{SC}_{11}(12)$ & 2 \\
\hline \multirow[t]{2}{*}{17} & $\mathrm{PS}_{17}(2)$ & 2 & & $\mathrm{SC}_{11}(60)$ & 2 \\
\hline & $\mathrm{PS}_{17}(4)$ & & 126 & $\mathrm{St}_{2}, \mathrm{SC}_{3}(4), \mathrm{St}_{7}$ & 2 \\
\hline \multirow[t]{2}{*}{18} & $\mathrm{St}_{2}, \mathrm{SC}_{3}(4)$ & $2,4,8$ & & $\mathrm{St}_{2}, \mathrm{SC}_{3}(8), \mathrm{PS}_{7}(2)$ & 2 \\
\hline & $\mathrm{St}_{2}, \mathrm{SC}_{3}(8)$ & 5 & 135 & $\mathrm{SC}_{27}(\sqrt{-3}, 1), \mathrm{St}_{5}$ & 2 \\
\hline 19 & $\mathrm{PS}_{19}(3)$ & 2 & 147 & $\mathrm{St}_{3}, \mathrm{SC}_{7}(4)$ & 2 \\
\hline 20 & $\mathrm{SC}_{2}(3), \mathrm{PS}_{5}(2)$ & 2 & & $\mathrm{St}_{3}, \mathrm{SC}_{7}(12)$ & 2 \\
\hline 22 & $\mathrm{St}_{2}, \mathrm{St}_{11}$ & 2 & 150 & $\mathrm{St}_{2}, \mathrm{St}_{3}, \mathrm{SC}_{5}(3)$ & 2 \\
\hline \multirow[t]{3}{*}{25} & $\mathrm{SC}_{5}(3)$ & 2 & & $\mathrm{St}_{2}, \mathrm{St}_{3}, \mathrm{SC}_{5}(6)$ & 2 \\
\hline & $\mathrm{SC}_{5}(6)$ & 2 & & $\mathrm{St}_{2}, \mathrm{St}_{3}, \mathrm{SC}_{5}(8)$ & 3 \\
\hline & $\mathrm{SC}_{5}(8)$ & 3 & & $\mathrm{St}_{2}, \mathrm{St}_{3}, \mathrm{SC}_{5}(12)$ & 12 \\
\hline
\end{tabular}




\begin{tabular}{|c|c|c|c|c|c|}
\hline Cond. & Local Components & $k$ & Cond. & Local Components & $k$ \\
\hline 162 & $\mathrm{St}_{2}, \mathrm{SC}_{81}$ & 2 & 578 & $\mathrm{St}_{2}, \mathrm{SC}_{17}(3)$ & \\
\hline 180 & $\mathrm{SC}_{2}(3), \mathrm{SC}_{3}(4), \mathrm{St}_{5}$ & 2 & & $\mathrm{St}_{2}, \mathrm{SC}_{17}(6)$ & \\
\hline & $\mathrm{SC}_{2}(3), \mathrm{SC}_{3}(4), \mathrm{PS}_{5}(2)$ & 2 & & $\mathrm{St}_{2}, \mathrm{SC}_{17}(12)$ & \\
\hline 192 & $\mathrm{SC}_{64}(1), \mathrm{St}_{3}$ & 2 & & $\mathrm{St}_{2}, \mathrm{SC}_{17}(24)$ & \\
\hline 196 & $\mathrm{SC}_{2}(3), \mathrm{SC}_{7}(4)$ & 2 & & $\mathrm{St}_{2}, \mathrm{SC}_{17}(48)$ & \\
\hline & $\mathrm{SC}_{2}(3), \mathrm{SC}_{7}(12)$ & 2 & 588 & $\mathrm{SC}_{2}(3), \mathrm{St}_{3}, \mathrm{SC}_{7}(8)$ & \\
\hline 225 & $\mathrm{SC}_{3}(8), \mathrm{SC}_{5}(8)$ & 2 & & $\mathrm{SC}_{2}(3), \mathrm{St}_{3}, \mathrm{SC}_{7}(24)$ & \\
\hline 234 & $\mathrm{St}_{2}, \mathrm{SC}_{3}(4), \mathrm{PS}_{13}(2)$ & 2 & 675 & $\mathrm{SC}_{27}(\sqrt{-3},-1), \mathrm{SC}_{5}(8)$ & \\
\hline 242 & $\mathrm{St}_{2}, \mathrm{SC}_{11}(4)$ & 2 & 726 & $\mathrm{St}_{2}, \mathrm{St}_{3}, \mathrm{SC}_{11}(12)$ & \\
\hline & $\mathrm{St}_{2}, \mathrm{SC}_{11}(20)$ & 2 & & $\mathrm{St}_{2}, \mathrm{St}_{3}, \mathrm{SC}_{11}(60)$ & \\
\hline 252 & $\mathrm{SC}_{2}(3), \mathrm{SC}_{3}(4), \mathrm{St}_{7}$ & 2 & 882 & $\mathrm{St}_{2}, \mathrm{SC}_{3}(4), \mathrm{SC}_{7}(4)$ & \\
\hline 256 & $\mathrm{SC}_{256}(0)$ & 2 & & $\mathrm{St}_{2}, \mathrm{SC}_{3}(4), \mathrm{SC}_{7}(12)$ & 2 \\
\hline 270 & $\mathrm{St}_{2}, \mathrm{SC}_{27}(\sqrt{3}, 1), \mathrm{St}_{5}$ & 2 & 900 & $\mathrm{SC}_{2}(3), \mathrm{SC}_{3}(8), \mathrm{SC}_{5}(8)$ & \\
\hline 294 & $\mathrm{St}_{2}, \mathrm{St}_{3}, \mathrm{SC}_{7}(8)$ & 2 & 1058 & $\mathrm{St}_{2}, \mathrm{SC}_{23}(4)$ & \\
\hline & $\mathrm{St}_{2}, \mathrm{St}_{3}, \mathrm{SC}_{7}(24)$ & 2 & & $\mathrm{St}_{2}, \mathrm{SC}_{23}(44)$ & \\
\hline 320 & $\mathrm{SC}_{64}(0), \mathrm{St}_{5}$ & 2 & 1089 & $\mathrm{SC}_{3}(4), \mathrm{SC}_{11}(4)$ & \\
\hline 324 & $\mathrm{SC}_{2}(3), \mathrm{SC}_{81}$ & 2 & & $\mathrm{SC}_{3}(4), \mathrm{SC}_{11}(20)$ & \\
\hline 350 & $\mathrm{St}_{2}, \mathrm{SC}_{5}(8), \mathrm{PS}_{7}(2)$ & 2 & 1350 & $\mathrm{St}_{2}, \mathrm{SC}_{27}(\sqrt{-3}, 1), \mathrm{SC}_{5}(3)$ & \\
\hline 378 & $\mathrm{St}_{2}, \mathrm{SC}_{27}(\sqrt{-3},-1), \mathrm{PS}_{7}(2)$ & 2 & & $\mathrm{St}_{2}, \mathrm{SC}_{27}(\sqrt{-3}, 1), \mathrm{SC}_{5}(6)$ & ' \\
\hline 396 & $\mathrm{SC}_{2}(3), \mathrm{SC}_{3}(4), \mathrm{St}_{11}$ & 2 & & $\mathrm{St}_{2}, \mathrm{SC}_{27}(\sqrt{-3}, 1), \mathrm{SC}_{5}(12)$ & 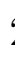 \\
\hline 441 & $\mathrm{SC}_{3}(4), \mathrm{SC}_{7}(8)$ & 2 & & $\mathrm{St}_{2}, \mathrm{SC}_{27}(\sqrt{-3},-1), \mathrm{SC}_{5}(24)$ & \\
\hline & $\mathrm{SC}_{3}(4), \mathrm{SC}_{7}(24)$ & 2 & & $\mathrm{St}_{2}, \mathrm{SC}_{27}(\sqrt{-3}, 1), \mathrm{SC}_{5}(8)$ & \\
\hline 450 & $\mathrm{St}_{2}, \mathrm{SC}_{3}(4), \mathrm{SC}_{5}(3)$ & 2 & 1452 & $\mathrm{SC}_{2}(3), \mathrm{St}_{3}, \mathrm{SC}_{11}(4)$ & \\
\hline & $\mathrm{St}_{2}, \mathrm{SC}_{3}(4), \mathrm{SC}_{5}(6)$ & 2 & & $\mathrm{SC}_{2}(3), \mathrm{St}_{3}, \mathrm{SC}_{11}(20)$ & \\
\hline & $\mathrm{St}_{2}, \mathrm{SC}_{3}(4), \mathrm{SC}_{5}(8)$ & 3 & 1600 & $\mathrm{SC}_{64}(1), \mathrm{SC}_{5}(8)$ & \\
\hline & $\mathrm{St}_{2}, \mathrm{SC}_{3}(4), \mathrm{SC}_{5}(12)$ & 2 & 1728 & $\mathrm{SC}_{64}(0), \mathrm{SC}_{27}(\sqrt{-3}, 1)$ & \\
\hline & $\mathrm{St}_{2}, \mathrm{SC}_{3}(8), \mathrm{SC}_{5}(24)$ & 2 & & $\mathrm{SC}_{64}(1), \mathrm{SC}_{27}(\sqrt{-3},-1)$ & \\
\hline 484 & $\mathrm{SC}_{2}(3), \mathrm{SC}_{11}(3)$ & 2 & 1764 & $\mathrm{SC}_{2}(3), \mathrm{SC}_{3}(4), \mathrm{SC}_{7}(4)$ & \\
\hline & $\mathrm{SC}_{2}(3), \mathrm{SC}_{11}(6)$ & 2 & & $\mathrm{SC}_{2}(3), \mathrm{SC}_{3}(4), \mathrm{SC}_{7}(12)$ & \\
\hline & $\mathrm{SC}_{2}(3), \mathrm{SC}_{11}(15)$ & 2 & 2178 & $\mathrm{St}_{2}, \mathrm{SC}_{3}(4), \mathrm{SC}_{11}(3)$ & \\
\hline & $\mathrm{SC}_{2}(3), \mathrm{SC}_{11}(30)$ & 2 & & $\mathrm{St}_{2}, \mathrm{SC}_{3}(4), \mathrm{SC}_{11}(6)$ & \\
\hline 540 & $\mathrm{SC}_{2}(3), \mathrm{SC}_{27}(\sqrt{-3}, 1), \mathrm{St}_{5}$ & 2 & & $\mathrm{St}_{2}, \mathrm{SC}_{3}(4), \mathrm{SC}_{11}(15)$ & \\
\hline 576 & $\begin{array}{l}\mathrm{SC}_{64}(1), \mathrm{SC}_{3}(4) \\
\mathrm{SC}_{64}(2), \mathrm{SC}_{3}(8)\end{array}$ & $\begin{array}{l}2 \\
2\end{array}$ & & $\mathrm{St}_{2}, \mathrm{SC}_{3}(4), \mathrm{SC}_{11}(30)$ & \\
\hline
\end{tabular}


TABLE 2. Explanation of symbols appearing as local types for $\mathbf{Q}_{p}$ in Table 1, listed with dimension and conductor.

\begin{tabular}{|c|c|c|c|}
\hline Symbol & Definition & Dimension & Conductor \\
\hline $\mathrm{St}_{p}$ & Steinberg representation of $\mathrm{GL}(2, \mathbf{Z} / p \mathbf{Z})$. & $p$ & $p$ \\
\hline $\mathrm{PS}_{p}(n)$ & $\begin{array}{l}\text { Principal Series rep. of } \mathrm{GL}(2, \mathbf{Z} / p \mathbf{Z}) \text { correspond- } \\
\text { ing to the characters } \varepsilon \text { and } 1 \text { of }(\mathbf{Z} / p \mathbf{Z})^{*} \text {, where } \varepsilon \\
\text { has order } n \text {. }\end{array}$ & $p+1$ & $p$ \\
\hline $\mathrm{SC}_{p}(n)$ & $\begin{array}{l}\text { Cuspidal rep. of } \mathrm{GL}(2, \mathbf{Z} / p \mathbf{Z}) \text { corresponding to a } \\
\text { multiplicative character } \theta \text { of order } n \text { of a quadratic } \\
\text { field extension of } \mathbf{Z} / p \mathbf{Z} \text {. }\end{array}$ & $p-1$ & $p^{2}$ \\
\hline $\mathrm{PS}_{4}$ & $\begin{array}{l}\text { Principal Series rep. of } \mathrm{GL}(2, \mathbf{Z} / 4 \mathbf{Z}) \text { correspond- } \\
\text { ing to the unique primitive character of conductor } \\
4 .\end{array}$ & 6 & 4 \\
\hline $\mathrm{PS}_{8}$ & $\begin{array}{l}\text { Principal Series rep. of } \mathrm{GL}(2, \mathbf{Z} / 8 \mathbf{Z}) \text { correspond- } \\
\text { ing to the unique even primitive character of con- } \\
\text { ductor } 8 \text {. }\end{array}$ & 12 & 8 \\
\hline $\mathrm{PS}_{9}$ & $\begin{array}{l}\text { Principal Series rep. of } \mathrm{GL}(2, \mathbf{Z} / 9 \mathbf{Z}) \text { correspond- } \\
\text { ing to any even primitive character of conductor } \\
9 .\end{array}$ & 12 & 9 \\
\hline $\mathrm{SC}_{8}$ & $\begin{array}{l}\text { Type belonging to a supercusp. rep. of } \mathrm{GL}\left(2, \mathbf{Q}_{2}\right) \\
\text { of conductor } 8 \text { with even central character. }\end{array}$ & 3 & 8 \\
\hline $\mathrm{SC}_{64}(n)$ & $\begin{array}{l}\text { Type belonging to a supercusp. rep. of } \operatorname{GL}\left(2, \mathbf{Q}_{2}\right) \\
\text { attached to any mult. character } \theta \text { of conductor } \\
8 \text { of the unramified extension } \mathbf{Q}_{2}(\rho) \text {, where } \rho \text { is } \\
\text { a primitive } 6 \text { th root of } 1 \text {; the value of } \theta(\rho) \text { is a } \\
\text { primitive } n \text {th root of } 1 \text {. }\end{array}$ & 4 & 64 \\
\hline $\mathrm{SC}_{256}(n)$ & $\begin{array}{l}\text { Type belonging to a supercusp. rep. of } \mathrm{GL}\left(2, \mathbf{Q}_{2}\right) \\
\text { attached to a mult. character } \theta \text { of conductor } 16 \text { of } \\
\text { the unramified extension } \mathbf{Q}_{2}(\rho) \text {; the value of } \theta(\rho) \\
\text { is a primitive } n \text {th root of } 1 \text {. }\end{array}$ & 8 & 256 \\
\hline $\mathrm{SC}_{27}(\sqrt{ \pm 3}, \iota)$ & $\begin{array}{l}\text { Type belonging to a supercusp. rep. } \pi \text { of } \\
\text { GL }\left(2, \mathbf{Q}_{3}\right) \text { attached to a character } \theta \text { of conductor } \\
9 \text { of } \mathbf{Q}_{3}(\sqrt{ \pm 3})^{*} \text {, assuming that the central charac- } \\
\text { ter of } \pi \text { has sign } \iota \text {. This translates to the condition } \\
\theta(-1)=-\iota \text {. }\end{array}$ & 8 & 27 \\
\hline $\mathrm{SC}_{81}$ & $\begin{array}{l}\text { Type belonging to any supercusp. rep. of } \pi \text { of } \\
\text { conductor } 81 \text { whose central character is even. }\end{array}$ & 54 & 81 \\
\hline
\end{tabular}


Theorem 5.1. $J$ is semi-stable over $M$. Conversely, if $p^{n}$ is any odd prime power other than 3,5,7,9,11,13,17,19,27,49, or 121, then $M$ is the minimal extension of $\mathbf{Q}_{p}^{n r}$ over which $J$ becomes semi-stable.

Proof. The variety $J$ is isogenous to $\prod_{f} J_{f}$, where $f$ runs over Galois orbits of newforms of conductor dividing $p^{n}$, and where the $\ell$-adic Galois representation corresponding to $f$ arises from the $\ell$-adic Tate module of $J_{f}$. The abelian variety $J_{f}$ becomes semi-stable over an extension $L / \mathbf{Q}_{p}^{\mathrm{nr}}$ if an only if the local Weil-Deligne representation $\rho_{f, p}$ attached to $f$ at $p$ becomes unipotent when restricted to $\operatorname{Gal}\left(\overline{\mathbf{Q}}_{p} / L\right)$; see [Gro72], exp. IX. Since $\rho_{f, p}$ lies in $A_{n}$, Eq. 5.1 implies that $J_{f}$ must become semi-stable over the field $M$.

For the converse statement, suppose $J$ is semi-stable over $L \supset K^{\mathrm{nr}}$. Let $\rho_{p} \in A_{n}$ and assume that no twist of $\rho_{p}$ is unramified. Let $\pi_{p}$ be the admissible representation of $\mathrm{GL}\left(2, \mathbf{Q}_{p}\right)$ corresponding to $\rho_{p}$, and let $\tau_{p}=\tau\left(\pi_{p}\right)$ be its inertial type. Let $\tau \in \operatorname{Types}(\mathbf{Q})$ be a global type of weight 2 whose only nontrivial local component is $\tau_{p}$. The prime powers listed in the theorem are the only ones which appear as conductors in Table 1. Since $p^{n}$ is not among these, $S(\tau)$ contains a cusp form $f$. The assumption on $L$ then implies $W_{L} \subset \operatorname{ker} \rho_{p}$. Since $\rho_{p}$ was arbitrary, we may apply Eq. 5.1 to conclude that $L \supset M$, whence the theorem.

\section{REFERENCES}

[BW00] A. Borel and N. Wallach, Continuous cohomology, discrete subgroups, and representations of reductive groups, second ed., Mathematical Surveys and Monographs, vol. 67, American Mathematical Society, Providence, RI, 2000.

[Car83] H. Carayol, Sur les représentations $\ell$-adiques attachees aux formes modulaires de Hilbert, C. R. Acad. Sci. Paris. 296 (1983), no. 15, 629-632.

[Car86] Sur les représentations $\ell$-adiques associées aux formes modulaires de Hilbert, Annales scientifiques de l'É.N.S. 19 (1986), no. 3, 409468.

[Cas73] W. Casselman, The restriction of a representation of $G L_{2}(k)$ to $G L_{2}(\mathfrak{O})$, Mathematischen Annalen 206 (1973), no. 4, 311-318.

[Che07] G. Chenevier, On number fields with given ramification, Compositio Mathematica 143 (2007), no. 6, 1359-1373.

[Clo86] L. Clozel, On limit multiplicities of discrete series representations in spaces of automorphic forms, Inventiones Mathematicae 83 (1986), 265284.

[CO77] H. Cohen and J. Oesterlé, Dimensions des espaces de formes modulaires, Modular functions of one variable, VI (Proc. Second Internat. Conf., Univ. Bonn, Bonn, 1976), Springer, Berlin, 1977, pp. 69-78. Lecture Notes in Math., Vol. 627.

[DW78] D. DeGeorge and N. Wallach, Limit formulas for multiplicities in $L^{2}(\Gamma \backslash G)$, Ann. Math. 107 (1978), 133-150. 
[Gro72] A. Grothendieck, Groupes de monodromie en géométrie algébrique. I, Lecture Notes in Mathematics, Vol. 288, Springer-Verlag, Berlin, 1972, Séminaire de Géométrie Algébrique du Bois-Marie 1967-1969 (SGA 7 I), Dirigé par A. Grothendieck. Avec la collaboration de M. Raynaud et D. S. Rim.

[Hen02] G. Henniart, Sur l'unicité des types pour $G L(2)$., Duke Math. J. 155 (2002), no. 2, 205-310.

[JL70] H. Jacquet and R. P. Langlands, Automorphic forms on GL(2), Lecture Notes in Mathematics, Vol. 114, Springer-Verlag, Berlin, 1970.

[KP96] C.S. Khare and D. Prasad, Extending local representations to global representations, Kyoto J. of Math. 36 (1996), 471-480.

[Kri96] M. Krir, Degré d'une extension de $\mathbf{Q}_{p}^{n r}$ sur laquelle $J_{0}(N)$ est semi-stable, Annales de l'institut Fourier 2 (1996), no. 46, 279-291.

[Kut78a] P. C. Kutzko, On the supercuspidal representations of $\mathrm{Gl}_{2}$, Amer. J. Math. 100 (1978), no. 1, 43-60.

[Kut78b] , On the supercuspidal representations of $\mathrm{Gl}_{2}$. II, Amer. J. Math. 100 (1978), no. 4, 705-716.

[Kut80] P.C. Kutzko, The Langlands conjecture for $G L_{2}$ of a local field, Annals of Math. 112 (1980), 381-412.

[Lan73] R. P. Langlands, Modular forms and $\ell$-adic representations, Modular functions of one variable, II (Proc. Internat. Summer School, Univ. Antwerp, Antwerp, 1972), Springer, Berlin, 1973, pp. 361-500. Lecture Notes in Math., Vol. 349. MR MR0354617 (50 \#7095)

[Oht83] Masami Ohta, On the zeta function of an abelian scheme over the Shimura curve, Japan. J. Math. (N.S.) 9 (1983), no. 1, 1-25.

[RT83] J. D. Rogawski and J. B. Tunnell, On Artin L-functions associated to Hilbert modular forms of weight one, Invent. Math. 74 (1983), no. 1, $1-42$.

[Shi71] G. Shimura, Introduction to the arithmetic theory of automorphic forms, Princeton University Press, 1971.

[Tat79] J. Tate, Number theoretic background, Proc. Symp. Pure Math. 33, part 2 (1979), 3-26.

[Tay89] R. Taylor, On galois representations associated to hilbert modular forms, Inventiones Mathematicae 98 (1989), 265-280.

[Vig80] M.-F. Vignéras, Arithmétique des algebres de quaternions, Lecture Notes in Mathematics, vol. 800, Springer-Verlag, 1980.

E-mail address: jared@math.ucla.edu

UCLA Department of Mathematics, Box 951555 Los Angeles, CA 90095-1555 USA 